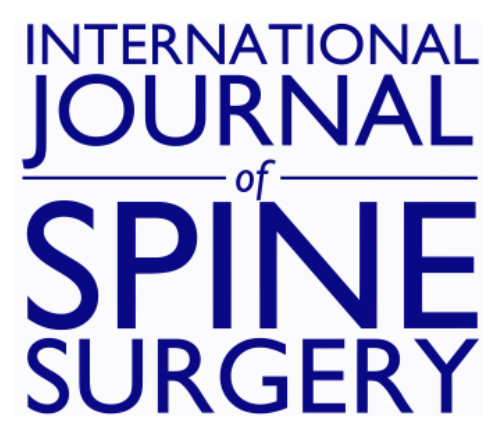

\title{
Review of Vertebral Augmentation: An Updated Meta-analysis of the Effectiveness
}

\author{
DOUGLAS BEALL, MORGAN P. LORIO, B. MIN YUN, MARIA J. RUNA, KEVIN L. ONG \\ and CHRISTOPHER B. WARNER
}

Int J Spine Surg 2018, 12 (3) 295-321

doi: https://doi.org/10.14444/5036

http://ijssurgery.com/content/12/3/295

This information is current as of April 26, 2023.

Email Alerts Receive free email-alerts when new articles cite this article. Sign up at: http://ijssurgery.com/alerts 


\title{
Review of Vertebral Augmentation: An Updated Meta- analysis of the Effectiveness
}

\author{
DOUGLAS BEALL, MD, ${ }^{1}$ MORGAN P. LORIO, MD ${ }^{2}$ B. MIN YUN, PHD, ${ }^{3}$ MARIA J. RUNA, PHD, ${ }^{3}$ KEVIN \\ L. ONG, PHD, PE, ${ }^{3}$ CHRISTOPHER B. WARNER, MD ${ }^{4}$

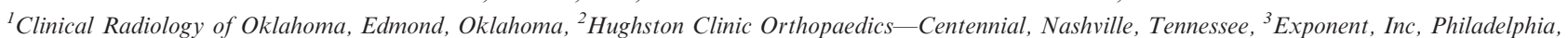 \\ Pennsylvania, ${ }^{4}$ University of Colorado Anschutz Medical Campus, Department of Radiology, Aurora, Colorado
}

\begin{abstract}
Background: To update vertebral augmentation literature by comparing outcomes between vertebroplasty (VP), balloon kyphoplasty (BKP), vertebral augmentation with implant (VAI), and nonsurgical management (NSM) for treating vertebral compression fractures (VCFs).

Methods: A PubMed literature search was conducted with keywords kyphoplasty, vertebroplasty, vertebral body stent, and vertebral augmentation AND implant for English-language articles from February 1, 2011, to November 22, 2016. Among the results, 25 met the inclusion criteria for the meta-analysis. Inclusion criteria were prospective comparative studies for mid-/lower-thoracic and lumbar VCFs enrolling at least 20 patients. Exclusion criteria included studies that were single arm, systematic reviews and meta-analyses, traumatic nonosteoporotic or cancer-related fractures, lack of clinical outcomes, or non-Level I and non-Level II studies. Standardized mean difference between baseline and end point for each outcome was calculated, and treatment groups were pooled using random effects meta-analysis.

Results: Visual analog scale pain reduction for BKP and VP was -4.05 and -3.88 , respectively. VP was better than but not significantly different from NSM (-2.66), yet BKP showed significant improvement from both NSM and VAI (-2.77). The Oswestry Disability Index reduction for BKP showed a significant improvement over VAI $(P<.001)$. There was no significant difference in changes between BKP and VP for anterior $(P=.226)$ and posterior $(P=.293)$ vertebral height restoration. There was no significant difference in subsequent fractures following BKP $(32.7 \%$; $95 \%$ confidence interval [CI]: $8.8 \%-56.6 \%)$ or VP $(28.3 \% ; 95 \%$ CI: $7.0 \%-49.7 \%)$ compared with NSM $(15.9 \% ; 95 \%$ CI: $5.2 \%-26.6 \%)$.

Conclusions/Level of Evidence: Based on Level I and II studies, BKP had significantly better and VP tended to have better pain reduction compared with NSM. BKP tended to have better height restoration than VP. Additionally, BKP had significant improvements in pain reduction and disability score as compared with VAI.

Clinical Relevance: This meta-analysis serves to further define and support the safety and efficacy of vertebral augmentation.
\end{abstract}

Other \& Special Categories

Keywords: meta-analysis, vertebral augmentation, vertebral compression fractures, vertebroplasty, balloon kyphoplasty

\section{INTRODUCTION}

Vertebral compression fractures (VCFs) are costly and are becoming even more common as more than 10000 Americans turn 65 years old each day. In the United States, there are 1.5 million VCFs annually, and worldwide a vertebral fracture occurs every 22 seconds. ${ }^{1-3}$ Symptomatic fractures usually present with sudden onset of back pain and functional debilitation in an elderly patient with osteoporosis, though many fractures may be asymptomatic. VCFs are expensive to treat, costing around $\$ 17$ billion per year. ${ }^{4,5}$ Morbidities associated with VCFs are substantial and can result in permanent loss of mobility and quality of life and lead to substantial disability. ${ }^{6}$
In addition, the deconditioning that affects patients with VCFs leads to mortality at a far higher rate than in age-matched controls. ${ }^{7,8}$ Increased mortality associated with VCFs has been well established for quite some time, but effects on mortality when patients undergo treatment with vertebral augmentation has only been described recently. ${ }^{9-12}$ Edidin et $\mathrm{al}^{13}$ reported significant reduction in morbidity and mortality in over a million patients with VCFs treated with vertebral augmentation as compared with patients treated with nonsurgical management (NSM).

Vertebral augmentation, including kyphoplasty and vertebroplasty (VP), have been accepted treatments for VCFs for decades. Balloon kyphoplasty 
(BKP) has had a large body of data supporting its use since receiving $510(\mathrm{k})$ clearance in 1998. More recently, interventionalists have started using implants and vertebral body stenting (VBS) in vertebral augmentation. One such implant is Kiva (Benvenue Medical, Inc, Santa Clara, California), a polyetheretherketone implant placed over a nitinol wire. Kiva was approved by the US Food and Drug Administration in 2014 after a randomized controlled trial (RCT) showed noninferiority to BKP, while maintaining an optimal safety profile and significantly improving patients' pain and function. ${ }^{16}$ VBS uses an expandable metal stent to restore vertebral height and currently is only available in Europe. Level I evidence concludes that although VBS is noninferior to BKP, in terms of patient outcomes, it is associated with a higher number of material related complications. ${ }^{15}$ The SpineJack (Vexim, SA, Balma, France), $\mathrm{CE}$ marked for use in Europe and other countries around the world, showed superiority to BKP in restoring $\mathrm{VCF}$ heights in a single-center trial ${ }^{16}$ and in a cadaver study. ${ }^{17}$ SpineJack is currently undergoing a comparison study with BKP in Europe and may be approved for use in the United States as early as 2018.

In 2009, 5 major societies developed a consensus statement on percutaneous vertebral augmentation, ${ }^{18}$ concluding that "percutaneous vertebral augmentation with vertebroplasty and kyphoplasty is a safe, efficacious, and durable procedure in appropriate patients with symptomatic osteoporotic and neoplastic fractures when performed in a manner in accordance with published standards." Also in 2009, 2 randomized trials on VP as treatment for osteoporotic vertebral fractures (Buchbinder et $\mathrm{al}^{19}$ and Kallmes et $\mathrm{al}^{20}$ ) were published in the New England Journal of Medicine (NEJM). These studies found no significant difference between vertebroplasty and sham treatment and prompted a debate on the effectiveness of surgical treatment of VCFs, as well as numerous changes in clinical recommendations and adverse decisions on procedure reimbursement. Later, 2 blinded RCTs demonstrated statistically significant benefits in pain improvement and functional improvement of vertebroplasty when compared with sham treatments. ${ }^{21,22}$ The impact of these studies is being evaluated concurrently.

The purpose of this meta-analysis was to update the existing body of literature using recent highestquality data to assess the effectiveness of BKP and VP, including vertebral augmenation with implant, compared with NSM in the treatment of patients with painful VCFs. Intertreatment analysis was also peformed. This meta-analysis also provided an updated review to guide an evidence-based approach to the use of vertebral augmentation procedures. This will provide an organizational framework to better define the heterogenous body of vertebral augmentation literature by analyzing the newest Level I and Level II studies.

\section{MATERIALS AND METHODS}

\section{Information Source}

PubMed, for articles published from February 1, 2011, to November 22, 2016.

\section{Search}

A PubMed search was performed with the assistance of a research librarian using keywords kyphoplasty, vertebroplasty, vertebral body stent, and vertebral augmentation AND implant for articles published in the English language from February 1, 2011, to November 22, 2016, resulting in 937 articles. The electronic search strings used by the research librarian to perform this PubMed search are included in Appendix I.

\section{Eligibility Criteria and Study Selection}

Inclusion criteria were as follows: prospective comparative studies of vertebral augmentation procedures, studies enrolling at least 20 patients, and studies performed for mid-/lower-thoracic and lumbar vertebral fractures (T5 through L5) due to osteoporosis.

Exclusion criteria were as follows: single-arm studies, kyphoplasty studies not using inflatable balloons, non-English language studies, systematic reviews and meta-analyses, traumatic nonosteoporotic or cancer-related fracture studies, studies without clinical outcomes, non-Level I and nonLevel II studies, and studies involving sacroplasty. Vertebral body stenting and KIVA procedures were grouped as a separate treatment group (vertebral augmentation with implants, or "VAI"). ${ }^{14,23-25}$

\section{Review Protocol and Data Collection Process}

A systematic review protocol was established to determine which papers satisfied the inclusion/ exclusion criteria and qualified for the meta-analysis. Abstracts for the 937 articles were reviewed by each 
of 2 reviewers to identify those that failed to meet the inclusion/exclusion criteria. For studies in which the abstracts were not adequate to reach a determination for inclusion/exclusion, the articles were reviewed for further assessment. Any discrepancies for qualification for the meta-analysis between the 2 reviewers were discussed and resolved together. After systematic review, 28 of these studies satisfied the inclusion/exclusion criteria. Follow-up length for these studies ranged from 6 months to 5 years.

\section{Data Items}

Outcomes of interest for this meta-analysis were as follows:

- Optimal intervention time/age of compression fracture

- Cost/benefit of surgical intervention versus NSM

- Economic considerations

- Quality of life (QOL) improvement as measured by the Short-Form 36 Survey Physical Component Summary (SF-36 PCS), SF-36, EuroQol-5D (EQ-5D), Quality of Life Questionnaire of the European Foundation for Osteoporosis (QUALEFFO), and low-back pain

- Disability improvement as measured by the Roland Morris Disability Questionnaire (RMDQ) score, disability days, Oswestry Disability Index (ODI), and the time up-andgo (TUG) metric

- Pain scores (10-point back pain, SF-36 bodily pain, visual analog scale [VAS], numeric rating scale, numerical visual scale)

- Subsequent adjacent fractures and overall subsequent fractures

- Cement extravasation

- Spinal canal extravasation

- Vertebral height restoration (anterior, midline, posterior)

- Kyphotic angle

- Serious adverse events (complications)

\section{STATISTICAL METHODS}

\section{Data Collection Process}

Data was extracted from each study from the provided tables and figures as well as the text in the articles. Extracted data was confirmed for accuracy by 2 reviewers. For each study, the standardized mean difference between baseline and endpoint for each outcome was calculated, as the scale for some outcomes varied across studies. For outcomes measured over time, the last time observed was considered the endpoint.

\section{Synthesis of Results}

Treatment groups were pooled using random effects meta-analysis to provide an overall estimate of effect. Pairwise comparisons were implemented using the $z$ test. ${ }^{26}$ Summary statistics were used to calculate mean and standard deviation if unknown. ${ }^{27}$ To assess heterogeneity, $\mathrm{I}^{2}$ statistics were calculated for each summary effect size. ${ }^{27} \mathrm{~A}$ minimum of 3 studies in each treatment group were required to perform analysis to estimate a withingroup effect. Mean effect sizes with $95 \%$ confidence intervals in parentheses are reported. The analysis was completed in SAS v.9.4 (SAS Inc, Cary, North Carolina).

\section{Summary Measures}

In addition to applying the random effects model for a pooled estimate, response ratios of standardized means between treatment groups within studies were calculated for outcomes of interest. This only included studies that had specific outcome measures for both treatment groups that were being compared.

\section{RESULTS}

Of the 28 studies, 3 did not report any outcomes of interest and were excluded, leaving 25 studies for meta-analysis. Six studies compared BKP to NSM (4 randomized versus 2 nonrandomized). Six studies compared VP to NSM (5 randomized versus 1 nonrandomized). Nine studies compared BKP to VP (5 randomized versus 4 nonrandomized). Four studies compared BKP to VAI (3 randomized versus 1 nonrandomized). Of the 25 studies included in the meta-analysis, 10 were Level I studies and 15 were Level II studies, based on the American Academy of Orthopaedic Surgeons guidelines for level of evidence. ${ }^{28}$ Due to the requirement of 3 studies to estimate within-group effect, some outcomes of interest (optimal intervention time/age of fracture, cost/benefit analysis, economic considerations, cement extravasation, spinal canal extravasation, and serious adverse events) could not be reported, as the minimum number of studies was not reached. Table 1 summarizes the findings of each study included in 
Table 1. Summary of study findings.

\begin{tabular}{|c|c|c|c|}
\hline $\begin{array}{l}\text { Author/Year of } \\
\text { Publication }\end{array}$ & Baseline Characteristics & Pain Relief & Disability \\
\hline Boonen et $\mathrm{al}^{28}$ & $\begin{array}{l}\text { 1. BKP ( } 149 \text { patients, average age } \\
72.2 \text { y) versus NSM }(151 \\
\text { patients, average age } 74.1 \mathrm{y}) \text { at } \\
24 \text { mo. } \\
\text { 2. Acute No. } \\
\text { 3. Baseline/screening, } 1,3,6,12 \text {, } \\
24 \text { mo. Back pain also at } 7 \mathrm{~d} \text {. } \\
\text { Randomized, nonblinded trial. } \\
\text { Level II study. }\end{array}$ & $\begin{array}{l}\text { 10-point back pain: } \\
\text { BKP superior to NSM }(-1.49, \\
P<.0001) \text { over } 24 \text { mo. BKP } \\
\text { superior to NSM at all time } \\
\text { points }(\mathrm{SS}) \text {. } \\
\text { SF-36 subscale for bodily pain: } \\
\text { BKP superior to NSM ( } 9.75 \\
\text { points, } P<.0001) \text { over } 24 \text { mo. } \\
\text { BKP superior to NSM at all } \\
\text { time points (SS). }\end{array}$ & $\begin{array}{l}\text { 24-point RMDQ: } \\
\text { BKP superior to NSM }(-3.01, \\
P<.0001) \text { over } 24 \text { mo. } \\
\text { BKP superior to NSM }(-2.81, P \\
=.0001) \text { at } 12 \mathrm{mo} \text {, superior by } \\
-1.43 \text { points, but NSS }(P \\
=.051) \text { at } 24 \text { mo. } \\
\text { Disability days: } \\
\text { BKP superior to NSM }(2.62 \mathrm{~d} \text {, } \\
P<.0001) \text { over } 24 \text { mo, SS at } \\
12 \text { mo }(-2.04, P=.009), \mathrm{NSS} \\
\text { at } 24 \text { mo }\end{array}$ \\
\hline Borgström et $\mathrm{al}^{26}$ & $\begin{array}{l}\text { 1. BKP (149 patients, average age } \\
71.7 \text { y) versus NSM }(151 \\
\text { patients, average age } 73.6 \mathrm{y}) \\
\text { 2. Acute No. } \\
\text { 3. Baseline/screening, } 1,3,6,12 \text {, } \\
24 \text { mo. Back pain at } 7 \mathrm{~d} \text {. } \\
\text { Randomized, nonblinded trial. } \\
\text { Level II study. }\end{array}$ & $\begin{array}{l}60 \% \text { of comparative gain of BKP } \\
\text { is driven by improvements in } \\
\text { pain. } \\
\text { VAS: } \\
\text { BKP superior to NSM }(-2.12 \\
\text { points, } P=.00 \text { ) over } 24 \text { mo. }\end{array}$ & $\begin{array}{l}\text { RMDQ: } \\
\text { BKP superior to NSM }(-3.42, P \\
\quad=.02) \text { over } 24 \text { mo. }\end{array}$ \\
\hline Chen et $\mathrm{al}^{9}$ & $\begin{array}{l}\text { 1. Total No. patients: } 96 \text {, from } \\
\text { Jan } 2007 \text { to Dec } 2012 \text {. } \\
\text { 2. PVP ( } 46 \text { patients, } 69 \text { vertebral } \\
\text { bodies, average age } 64.63 \text { y) } \\
\text { versus CT ( } 43 \text { patients, average } \\
\text { age } 66.49 \text { y). } \\
\text { 3. Baseline, 1d, } 1 \text { wk, } 1,3,6 \text { and } \\
12 \text { mo follow-up. } \\
\text { Level I study. }\end{array}$ & $\begin{array}{l}\text { VAS: } \\
\text { At baseline PVP similar to CT. } \\
\text { Improved pain relief after } 1 \mathrm{wk}, 1 \\
\text { mo, } 3 \text { mo, } 6 \text { mo, and } 1 \mathrm{y} \text { of } \\
\text { PVP. } \\
\text { Complete pain relief at final } \\
\text { follow-up higher in PVP (SS). } \\
\text { Use of analgesics after PVP } \\
\text { reduced after } 1 \mathrm{wk}, 1 \mathrm{mo}, 3 \mathrm{mo} \text {, } \\
6 \text { mo, } 1 \text { y (SS). }\end{array}$ & $\begin{array}{l}\text { ODI: } \\
\text { improved after } 1 \mathrm{wk}, 1 \mathrm{mo}, 3 \mathrm{mo} \text {, } \\
\quad 6 \mathrm{mo} \text {, and } 1 \text { y of PVP (SS). }\end{array}$ \\
\hline Clark et $\mathrm{al}^{21}$ & $\begin{array}{l}\text { 1. Total No. patients: } 120 \text {. } \\
\text { 2. Placebo ( } 59 \text { patients) versus VP } \\
\text { ( } 61 \text { patients). } \\
\text { 3. Follow-up: baseline, } 3 \mathrm{~d}, 14 \mathrm{~d} \text {, } \\
1 \text { mo, } 3 \text { mo, } 6 \text { mo. } \\
\text { Level I study. }\end{array}$ & $\begin{array}{l}\text { NRS score reduction: } \\
\text { Placebo: }-4.8 \\
\text { VP: }-6.1 \\
P=.043 \text { (SS, VP versus placebo) } \\
\text { VAS reduction: } \\
\text { Placebo: }-48 \\
\text { VP: }-58 \\
P=.050 \text { (NSS, VP versus } \\
\text { placebo) }\end{array}$ & $\begin{array}{l}\text { RMDQ reduction: } \\
\text { Placebo: }-7.4 \\
\text { VP: }-11.7 \\
P=.0022 \text { (SS, VP versus placebo) } \\
\text { TUG (baseline): } \\
\text { Placebo: } 29 \\
\text { VP: } 26\end{array}$ \\
\hline Dohm et $\mathrm{al}^{29}$ & $\begin{array}{l}\text { 1. BKP (191 patients, average age } \\
75.5 \text { y) versus VP (190 patients, } \\
\text { average age } 75.7 \text { y) } \\
\text { 2. Acute No. } \\
\text { 3. Baseline/screening } 1,3,12,24 \\
\text { mo. Back pain at } 7 \mathrm{~d} \text {. } \\
\text { Randomized, nonblinded trial. } \\
\text { Level II study (follow-up at final } \\
\text { time point }<80 \% \text { ). }\end{array}$ & $\begin{array}{l}\text { BKP and VP both }-4.0 \text { for back } \\
\text { pain at } 24 \text { mo, NSS across } \\
\text { treatments. }\end{array}$ & $\begin{array}{l}\text { BKP }-26.9 \text { and VP }-25.9 \text { for } \\
\text { ODI at } 24 \text { mo. NSS across } \\
\text { treatments. }\end{array}$ \\
\hline Dong et $\mathrm{al}^{30}$ & $\begin{array}{l}\text { 1. BKP ( } 51 \text { patients, average age } \\
69.8 \text { y) versus VP ( } 35 \text { patients, } \\
\text { average age } 70.5 \text { y). } \\
\text { 2. "Gradual collapse of the } \\
\text { vertebral body." } \\
\text { 3. Follow-up of } 7 \text { to } 36 \text { mo } \\
\text { (average: } 21.3 \text { mo). } \\
\text { Level II study. }\end{array}$ & $\begin{array}{l}\text { VAS: } \\
\text { BKP and VP both decreased pain } \\
\text { scores (SS) after operation. No } \\
\text { comment made on difference } \\
\text { across procedures. }\end{array}$ & NR \\
\hline Endres and Badura ${ }^{23}$ & $\begin{array}{l}\text { 1. Total No. patients: } 59 \text {. } \\
\text { 2. BKP ( } 20 \text { patients, average age } \\
63.3 \text { y) versus VP ( } 21 \text { patients, } \\
\text { average age } 71.3 \text { y) versus SKP } \\
\text { ( } 18 \text { patients, average age } 67.1 \\
\text { y). } \\
\text { 3. Presurgery, directly } \\
\text { postsurgery, and } 6 \text { mo } \\
\text { postsurgery. } \\
\text { Level II study. }\end{array}$ & $\begin{array}{l}\text { VAS: } \\
\text { NSS between groups, at } \\
\text { presurgery and } 6 \text { mo } \\
\text { postsurgery. }\end{array}$ & $\begin{array}{l}\text { ODI: } \\
\text { NSS between groups, } \\
\text { at presurgery and } 6 \text { mo } \\
\text { postsurgery. }\end{array}$ \\
\hline
\end{tabular}


Table 1. Extended.

\begin{tabular}{|c|c|c|c|}
\hline Quality of Life & Kyphotic Angle (Grades) & Vertebral Height & Cement Extravasation \\
\hline $\begin{array}{l}\text { PCS: BKP superior to NSM }(+3.24, P \\
=.0004) \text { over } 24 \text { mo. SS at } 6 \text { mo but not at } \\
12 \text { or } 24 \text { mo. At } 24 \text { mo, BKP superior } \\
(+1.68, \mathrm{NSS} P=.15) \text {. } \\
\text { EQ-5D: BKP superior to NSM }(+0.12 \text { points, } \\
P=.0002) \text { over } 24 \text { mo. BKP superior to } \\
\text { NSM at all time points (SS). }\end{array}$ & NR & NR & $\begin{array}{l}\text { Compression fracture } \\
\text { serious adverse event } \\
\text { showed cement } \\
\text { migrated anteriorly }\end{array}$ \\
\hline $\begin{array}{l}\text { BKP gained on average more than NSM } \\
\text { patients for all instruments }(P<.05) \text {. } \\
\text { EQ-5D: BKP superior to NSM }(+0.20, P \\
\quad=.00) \text { over } 24 \text { mo. } \\
\text { SF-36: BKP superior to NSM }(+0.11, P \\
\quad=.03) \text { over } 24 \text { mo. }\end{array}$ & NR & NR & NR \\
\hline $\begin{array}{l}\text { RMDQ: } \\
\text { improved after } 1 \mathrm{wk}, 1 \mathrm{mo}, 3 \mathrm{mo}, 6 \mathrm{mo} \text {, and } \\
\quad 1 \text { y of PVP (SS). }\end{array}$ & NR & NR & $\begin{array}{l}\text { AP and lateral spinal } \\
\text { X-ray showed } \\
\text { leakage in } 36 \text { of } 69 \\
\text { vertebral bodies. }\end{array}$ \\
\hline $\begin{array}{l}\text { EQ-5D: } \\
\text { Placebo: } 0.74 \\
\text { VP: } 0.8 \\
P=.012 \text { (SS, VP versus placebo) } \\
\text { QUALEFFO: } \\
\text { Placebo: } 45 \\
\text { VP: } 38 \\
P=.032 \text { (SS, VP versus placebo) }\end{array}$ & NR & $\begin{array}{l}\text { Height loss: } \\
\text { VP: } 27 \% \\
\text { Placebo: } 63 \%\end{array}$ & $\begin{array}{l}\text { VP: Cement leakage at } \\
21 \text { of } 61 \text { patients } \\
(34 \%)\end{array}$ \\
\hline $\begin{array}{l}\text { BKP }+7.6 \text { and VP }+7.5 \text { for SF-36 PCS at } 24 \\
\text { mo. NSS across treatments. } \\
\text { BKP }+0.28 \text { and VP }+0.31 \text { for EQ-5D at } 24 \\
\text { mo. NSS across treatments. }\end{array}$ & $\begin{array}{l}\text { BKP superior to VP by } 1.42^{\circ} \text { at } \\
24 \mathrm{mo}, P=.036 \text {. }\end{array}$ & NR & $\begin{array}{l}\text { BKP lower }(P=.047) \\
\text { than VP for cement } \\
\text { extravasation. }\end{array}$ \\
\hline NR & $\begin{array}{l}\text { Mean improvement in local } \\
\text { kyphotic angle was } 2.31^{\circ} \text { and } \\
8.32^{\circ} \text { after VP and BKP, } \\
\text { respectively (SS). } \\
\text { BKP superior to VP in differences } \\
\text { of improvement (SS). }\end{array}$ & $\begin{array}{l}\text { VP: Restoration of anterior/ } \\
\text { posterior height was } 1.93 \mathrm{~mm} \\
\text { and } 0.47 \mathrm{~mm} \text {, respectively (SS). } \\
\text { BKP: Mean restoration of } \\
\text { anterior/posterior height was } \\
5.44 \mathrm{~mm} \text { and } 1.36 \mathrm{~mm} \text {, } \\
\text { respectively (SS). } \\
\text { BKP superior to VP in differences } \\
\text { of restoration of vertebral } \\
\text { height }(P<.001)\end{array}$ & NR \\
\hline NR & NR & $\begin{array}{l}\text { None of the groups improved } \\
\text { vertebral body height in the } \\
\text { anterior and central portion. }\end{array}$ & $\begin{array}{l}\text { Measured directly } \\
\text { postsurgery and } 6 \text { mo } \\
\text { postsurgery. } \\
\text { NSS differences: } \\
\text { VP: } 4 \text { lateral leakages } \\
\text { and } 4 \text { in the disk. } \\
\text { BKP: } 3 \text { laterals and } 1 \\
\text { anterior. } \\
\text { SKP: } 1 \text { in the disk. }\end{array}$ \\
\hline
\end{tabular}


Table 1. Continued. Extended.

\begin{tabular}{|c|c|c|c|}
\hline $\begin{array}{l}\text { Spinal Canal/ } \\
\text { Foramen } \\
\text { Extravasation }\end{array}$ & New VCF & Adverse Event & $\begin{array}{l}\text { Optimal } \\
\text { Intervention } \\
\quad \text { Time }\end{array}$ \\
\hline NR & $\begin{array}{l}\text { New fractures: no SS difference } \\
\text { ( } 56 \text { of } 118 \text { BKP fractures versus } \\
45 \text { of } 102 \text { NSM, } P=.68 \text { ). } \\
\text { No SS for adjacent radiographic } \\
\text { fractures, painful fractures. }\end{array}$ & $\begin{array}{l}11 \text { patients in BKP }(7.4 \%) \text { had } \\
\text { new clinical fractures } \\
\text { possibly caused by cement. } \\
\text { One serious AE from } \\
\text { recurrent compression } \\
\text { fracture. } \\
1 \text { hematoma and } 1 \\
\text { exacerbation of recurrent } \\
\text { UTI. } \\
\text { All deaths considered } \\
\text { unrelated to treatment ( } 23 \\
\text { total deaths, } 12 \\
\text { kyphoplasty, } 11 \mathrm{NSM}) \text {. }\end{array}$ & NR \\
\hline NR & NR & NR & NR \\
\hline NR & $\begin{array}{l}\text { NSS differences: } \\
3 \text { of } 46 \text { PVP patients versus } 7 \text { of } \\
43 \text { CT patients }\end{array}$ & NR & NR \\
\hline NR & $\begin{array}{l}\text { VP: } 3 \text { patients } \\
\text { Placebo: } 2 \text { patients }\end{array}$ & $\begin{array}{l}\text { VP: } 2 \text { patients (respiratory } \\
\text { arrest, humerus fracture) } \\
\text { Placebo: } 2 \text { patients (spinal } \\
\text { cord compression) }\end{array}$ & NR \\
\hline $\begin{array}{l}\text { BKP lower } \\
\text { intravascular } \\
\text { extravasation }(P \\
=.028) \text { than VP. }\end{array}$ & $\begin{array}{l}\text { BKP had } 8.6 \% \text { fewer } \\
\text { radiographic fractures versus } \\
\text { PVP }(P=.23) \text {. NSS } \\
\text { clinically identified fractures. BKP } \\
\text { had longer fracture-free survival } \\
(P=.0596), \text { NSS. }\end{array}$ & $\begin{array}{l}\text { Common: procedural pain, } \\
\text { back pain, new symptomatic } \\
\text { fracture. } \\
\text { Also: bronchitis, pneumonia, } \\
\text { UTI, etc. } \\
1 \text { cement embolism for BKP } \\
\text { and VP each. }\end{array}$ & NR \\
\hline NR & NR & NR & NR \\
\hline NR & $\begin{array}{l}\text { No adjacent fractures were } \\
\text { observed. }\end{array}$ & NR & NR \\
\hline
\end{tabular}


Table 1. Continued. Extended.

\begin{tabular}{|c|c|c|c|}
\hline $\begin{array}{c}\text { Cost/Benefit } \\
\text { of Surgical } \\
\text { Intervention }\end{array}$ & $\begin{array}{c}\text { Economic } \\
\text { Considerations }\end{array}$ & $\begin{array}{l}\text { QOL/SF-36 } \\
\text { for CABG/ } \\
\text { THA/TKA }\end{array}$ & Conclusion \\
\hline NR & NR & NR & $\begin{array}{l}\text { BKP has improved QOL, pain relief, disability } \\
\text { improvement, patient satisfaction for } 2 \text { y over } \\
\text { NSM. } \\
\text { Overall better performance of BKP over } 1 \text { y, } \\
\text { but patients improve around 2-y mark for } \\
\text { NSM. } \\
\text { BKP has improved disability (RMDQ) and } \\
\text { activity days at } 12 \text { mo but not at } 24 \text { mo. }\end{array}$ \\
\hline
\end{tabular}

NR

NR
BKP improves QOL, pain, disability compared to NSM over $2 \mathrm{y}$.

Pain relief shown to have most contribution to QOL measures/scales.

PVP was found to be associated with greater pain relief and improved functional outcomes at 1 y compared with $\mathrm{CT}$.

Vertebroplasty was shown to reduce pain from osteoporotic spinal fractures of less than $6 \mathrm{wk}$ when compared with a true placebo control.

BKP and VP have SS less pain, disability, QOL, but not SS between groups.

Both have similar AE profiles. Kyphoplasty had fewer cement leakages, a trend of longer fracture-free survival.

VP and BKP both improve pain scores, kyphotic angle, and vertebral heights. BKP superior to VP in improving kyphotic angle and vertebral height restoration.

Determined that the type of cement augmentation system used for primary osteoporosis patients does not matter. Overall, the vertebroplasty technique may be considered the surgical procedure of choice. 
Table 1. Continued

\begin{tabular}{|c|c|c|c|}
\hline $\begin{array}{l}\text { Author/Year of } \\
\text { Publication }\end{array}$ & Baseline Characteristics & Pain Relief & Disability \\
\hline Farrokhi et $\mathrm{al}^{31}$ & $\begin{array}{l}\text { 1. Total No. patients: } 82 \text {. } \\
\text { 2. PVP ( } 40 \text { patients, average age } \\
72 \text { y) versus OMT ( } 42 \text { patients, } \\
\text { average age } 74 \text { y), from Sep } \\
2004 \text { to Jan } 2006 \text {. } \\
\text { 3. Follow-up at } 1 \mathrm{wk}, 2,6,12,24 \text {, } \\
36 \text { mo postoperatively. } \\
\text { Level I study. }\end{array}$ & $\begin{array}{l}\text { VAS: } \\
\text { PVP reduced from } 8.4 \text { to } 1.8 \text {. } \\
\text { OMT reduced from } 7.2 \text { to } 3.7 \text {. } \\
\text { Better in PVP than OMT (SS at } 6 \\
\quad \text { mo, NSS at } 12-36 \text { mo). }\end{array}$ & NR \\
\hline Folman and Shabat ${ }^{32}$ & $\begin{array}{l}\text { 1. Total No. patients: } 45 \text {. } \\
\text { 2. CV ( } 14 \text { patients, average age } \\
75.6 \text { y) versus SK ( } 31 \text { patients, } \\
\text { average age } 70.74 \text { y). } \\
\text { 3. Follow-up at } 1,6 \text {, and } 12 \text { mo } \\
\text { after procedure. } \\
\text { Level II study. }\end{array}$ & $\begin{array}{l}\text { VAS: } \\
\text { Pretreatment and posttreatment } \\
\quad \text { NSS. } \\
\text { Reduced pain: } \\
\text { SK superior to CV. } \\
\text { Both SK and CV were SS in } \\
\text { reducing pain from pre-op. }\end{array}$ & NR \\
\hline Fritzell et $\mathrm{al}^{33}$ & $\begin{array}{l}\text { 1. Total No. patients: } 67 . \\
\text { 2. BKP ( } 35 \text { patients, average age } \\
72 \text { y) versus control }(32 \\
\text { patients, average age } 75 \mathrm{y}) \\
\text { 3. Acute/subacute }(<3 \mathrm{mo}) \text { No. } \\
\text { 4. Follow-up at } 1,3,6,12,24 \text { mo. } \\
\text { Level I study. }\end{array}$ & NR & NR \\
\hline Korovessis et $\mathrm{al}^{24}$ & $\begin{array}{l}\text { 1. Total No. patients: } 168 \text {. } \\
\text { 2. KIVA ( } 82 \text { patients, average age } \\
69 \text { y) versus BKP ( } 86 \text { patients, } \\
\text { average age } 72 \text { y) from May } \\
2010 \text { to Oct } 2011 \text {. } \\
\text { 3. Baseline, follow-up at } 14 \text { mo } \\
\text { postoperatively. } \\
\text { Level I study. }\end{array}$ & $\begin{array}{l}\text { VAS: } \\
\text { Improvement in both groups } \\
\text { postoperatively. } \\
\text { NSS between groups. }\end{array}$ & $\begin{array}{l}\text { ODI: } \\
\text { Improvement in both groups } \\
\text { postoperatively. } \\
\text { NSS between groups. }\end{array}$ \\
\hline Kroon et $\mathrm{al}^{34}$ & $\begin{array}{l}\text { 1. Total No. patients: } 57 \text {. } \\
\text { 2. VP ( } 29 \text { patients, average age } \\
76.7 \text { y) versus sham }(28 \\
\text { patients, average age } 77.7 \mathrm{y}) \\
\text { from Apr } 2004 \text { to Oct } 2008 \text {. } \\
\text { 3. Baseline, follow-up at } 12 \text { mo, } \\
24 \text { mo postoperatively. } \\
\text { Level II study. }\end{array}$ & $\begin{array}{l}\text { Overall pain: } \\
12 \text { mo: VP improved by } 2.4 \text { units } \\
\text { versus } 1.9 \text { units in sham. } \\
24 \text { mo: VP improved by } 3.0 \text { units } \\
\text { versus } 1.9 \text { units in sham. } \\
\text { No beneficial effects of VP over } \\
\text { sham. }\end{array}$ & $\begin{array}{l}\text { RMDQ: } \\
12 \text { mo: VP } 2.0 \text { units versus } 2.6 \\
\text { units in sham. } \\
24 \text { mo: VP } 2.6 \text { units versus } 2.7 \\
\text { units in sham. }\end{array}$ \\
\hline Lee et $\mathrm{al}^{35}$ & $\begin{array}{l}\text { 1. Total No. patients: } 231 \text {. } \\
\text { 2. CV-conservative treatment } \\
\text { (149 patients, average age } 66.2 \\
\text { y) versus BKP ( } 82 \text { patients, } \\
\text { average age } 76.8 \text { y) from Mar } \\
2005 \text { to May } 2009 \text {. } \\
\text { 3. Baseline, follow-up at } 1 \text { y } \\
\text { postoperatively. } \\
\text { Level II study. }\end{array}$ & $\begin{array}{l}\text { VAS: } \\
\text { Baseline similar and improved } \\
\text { after } 1 \text { y in both groups. } \\
\text { BKP only lower VAS up to } 1 \text { mo: } \\
3.2 \text { versus } 4.8 \text { in CV (SS). } \\
\text { Afterwards NSS. }\end{array}$ & $\begin{array}{l}\text { ODI: } \\
\text { Baseline similar and improved } \\
\text { after } 1 \text { y in both groups. } \\
\text { BKP only lower ODI up to } 1 \mathrm{mo} \text { : } \\
10.3 \text { versus } 17.08 \text { in CV (SS). } \\
\text { Afterwards NSS. }\end{array}$ \\
\hline Li et $\mathrm{al}^{36}$ & $\begin{array}{l}\text { 1. Total No. patients: } 85 \text {. } \\
\text { 2. BKP ( } 45 \text { patients, average age } \\
68.5 \text { y) versus VP ( } 40 \text { patients, } \\
\text { average age } 67.1 \text { y). } \\
\text { 3. Painful osteoporotic VCFs. } \\
\text { 4. Follow-up at } 3,6,12 \text { mo. } \\
\text { Level II study. }\end{array}$ & $\begin{array}{l}\text { VAS: } \\
\text { Both BKP and VP had } \\
\text { significantly (SS) less painful } \\
\text { scores } 1 \mathrm{~d} \text { after of surgery and } \\
12 \text { mo afterwards. There was no } \\
\text { SS difference between BKP and } \\
\text { VP. }\end{array}$ & $\begin{array}{l}\text { ODI: } \\
\text { Both BKP and VP had } \\
\text { significantly (SS) lower } \\
\text { disability score after } 3 \text { and } 12 \\
\text { mo. There was no SS difference } \\
\text { between BKP and VP. }\end{array}$ \\
\hline Liu et $\mathrm{al}^{37}$ & $\begin{array}{l}\text { 1. Total No. of patients: } 100 \text {. } \\
\text { 2. BKP ( } 50 \text { patients, average age } \\
72.3 \text { y) versus VP ( } 50 \text { patients, } \\
\text { average age } 74.3 \mathrm{y}) \\
\text { 3. Osteoporotic VCF. } \\
\text { 4. Follow-up period of } 5 \mathrm{y} \text {. } \\
\text { 5. Randomized controlled trial. } \\
\text { Level I study. }\end{array}$ & $\begin{array}{l}\text { VAS: } \\
\text { SS lower VAS scores after } 3 \mathrm{~d}, 1 \\
\text { y, } 2 \text { y, and } 5 \text { y for both BKP } \\
\text { and VP. No SS difference in } \\
\text { VAS scores for BKP versus VP. }\end{array}$ & NR \\
\hline
\end{tabular}


Table 1. Continued. Extended.

\begin{tabular}{|c|c|c|c|}
\hline Quality of Life & Kyphotic Angle (Grades) & Vertebral Height & Cement Extravasation \\
\hline $\begin{array}{l}\text { LBP: } \\
\text { PV reduced from } 52.2 \text { to } 8.0 \text {. } \\
\text { OMT reduced from } 50.4 \text { to } 22.0 \text {. } \\
\text { Better in PV than OMT (SS at all time } \\
\quad \text { points). }\end{array}$ & $\begin{array}{l}\text { SI: } \\
\text { PV reduced from } 20.0^{\circ} \text { to } 8.9^{\circ} \text {. } \\
\text { OMT increased from } 21.0^{\circ} \text { to } \\
23.0^{\circ} \text {. } \\
\text { PV superior to OMT (SS at all } \\
\text { time points). }\end{array}$ & $\begin{array}{l}\text { VBH: } \\
\text { PV increased from } 2.8 \text { to } 3.0 \text {. } \\
\text { OMT reduced from } 2.5 \text { to } 2.0 \text {. } \\
\text { PV restored VBH and prevented } \\
\text { spinal deformity versus OMT. } \\
\text { (SS at all time points) }\end{array}$ & $\begin{array}{l}\text { PV: epidural (1), discal } \\
\text { (5), paravertebral } \\
\text { space }(8)\end{array}$ \\
\hline NR & $\begin{array}{l}\text { Pretreatment and post treatment: } \\
\text { SK superior to } \mathrm{CV} \text {. }\end{array}$ & NR & NR \\
\hline $\begin{array}{l}\text { EQ-5D: } \\
\text { Improved significantly within both groups, } \\
\text { most significant within } 3 \text { mo. BKP had } \\
0.085 \text { difference compared with control over } \\
24 \text { mo (NSS). }\end{array}$ & NR & NR & $\begin{array}{l}\text { BKP patient, cement } \\
\text { in index vertebra } \\
\text { migrated toward } \\
\text { aorta in thoracic } \\
\text { region but without } \\
\text { clinical consequences. }\end{array}$ \\
\hline $\begin{array}{l}\text { SF-36: } \\
\text { Physical functioning and mental health } \\
\text { improved postoperatively. } \\
\text { NSS between groups. }\end{array}$ & $\begin{array}{l}\text { KIVA: Reduced Gardner } \\
\text { kyphotic angle. } 84 \% \text { of spines } \\
\text { had residual kyphosis }>5^{\circ} \\
\text { measured. } \\
\text { BKP: Reduced Gardner kyphotic } \\
\text { angle. } 100 \% \text { of spines had } \\
\text { residual kyphosis }>5^{\circ} \\
\text { measured. }\end{array}$ & $\begin{array}{l}\text { Anterior vertebral body height: } \\
\text { NSS. } \\
\text { Posterior vertebral body height: } \\
\text { restored by KIVA. } \\
\text { Midline vertebral body height: } \\
\text { NSS. }\end{array}$ & $\begin{array}{l}\text { KIVA: } 4 \text { patients } \\
\text { BKP: } 12 \text { patients }\end{array}$ \\
\hline $\begin{array}{l}\text { QUALEFFO: } \\
12 \text { mo: VP } 6.7 \text { units versus } 8.8 \text { units in sham. } \\
24 \text { mo: VP } 5.9 \text { units versus } 4.6 \text { units in sham. } \\
\text { QOL: } \\
12 \text { mo: VP } 0.1 \text { units versus } 0.2 \text { units in sham. } \\
24 \text { mo: VP } 0.1 \text { units versus } 0.1 \text { units in sham. } \\
\text { EQ-5D: } \\
12 \text { mo: VP } 0.2 \text { units versus } 0.2 \text { units in sham. } \\
24 \text { mo: VP } 0.2 \text { units versus } 0.2 \text { units in sham. } \\
\text { TUG: } \\
12 \text { mo: VP }-2.6 \text { s versus } 4.3 \mathrm{~s} \text { in sham. } \\
24 \text { mo: VP } 3.5 \mathrm{~s} \text { versus } 4.7 \mathrm{~s} \text { in sham. } \\
\text { NR }\end{array}$ & NR & NR & $\begin{array}{l}\text { Higher risk with higher } \\
\text { volume of injected } \\
\text { median cement. }\end{array}$ \\
\hline
\end{tabular}

NR

Both BKP and VP reduced kyphotic angle at follow-up of 12 mo. BKP had SS greater reduction $(P<.01)$ than $\mathrm{VP}$ group.

SS lower kyphotic wedge angles after $3 \mathrm{~d}, 1 \mathrm{y}, 2 \mathrm{y}$, and $5 \mathrm{y}$ for both BKP and VP.
Both BKP and VP had SS increase of mean vertebral heights after 12 mo. Higher degrees of height restoration were achieved in BKP versus VP (SS, $P<.05)$.

SS higher vertebral body height after $3 \mathrm{~d}, 1 \mathrm{y}, 2 \mathrm{y}$, and $5 \mathrm{y}$ for both BKP and VP.
BKP had 6 of 66 treated fractures with cement leaks, while VP had 18 of 52 with cement leaks.

Asymptomatic cement leakage in some cases. 
Table 1. Continued. Extended.

\begin{tabular}{|c|c|c|c|}
\hline $\begin{array}{l}\text { Spinal Canal/ } \\
\text { Foramen } \\
\text { Extravasation }\end{array}$ & New VF & Adverse Event & $\begin{array}{l}\text { Optimal } \\
\text { Intervention } \\
\text { Time }\end{array}$ \\
\hline NR & $\begin{array}{l}\text { PV: } 1 \text { patient } \\
\text { OMT: } 6 \text { patients }\end{array}$ & $\begin{array}{l}\text { Postoperative complications: } 1 \\
\text { patient with severe right } \\
\text { lower extremity pain and } \\
\text { weakness. }\end{array}$ & NR \\
\hline NR & NR & NR & NR \\
\hline NR & $\begin{array}{l}5 \text { BKP and } 4 \text { control patients had } \\
\text { new painful VCF in } 1 \text { or } 2 \\
\text { adjacent levels and were treated } \\
\text { either with new BKP or } \\
\text { continuous standard treatment. }\end{array}$ & $\begin{array}{l}1 \text { cement extravasation in BKP } \\
\text { group, } 1 \text { infection in BKP } \\
\text { group. }\end{array}$ & NR \\
\hline $\begin{array}{l}\text { KIVA: NR } \\
\text { BKP: } 2 \text { patients }\end{array}$ & $\begin{array}{l}\text { KIVA: } 10 \text { patients } \\
\text { BKP: } 11 \text { patients }\end{array}$ & NR & NR \\
\hline NR & $\begin{array}{l}\text { VP: } 14 \\
\text { Sham: } 13\end{array}$ & $\begin{array}{l}\text { New clinical fractures (hip, } \\
\text { ribs, pelvis, sternum, } \\
\text { shoulder, waist, elbow) up } \\
\text { to } 24 \text { mo. }\end{array}$ & NR \\
\hline NR & NR & NR & NR \\
\hline NR & $\begin{array}{l}9 \text { patients with BKP and } 7 \\
\text { patients with VP experienced } \\
\text { new fractures. }\end{array}$ & NR & NR \\
\hline NR & $\begin{array}{l}12 \text { BKP patients with new VCF, } \\
10 \text { VP patients with new VCF. } \\
\text { No SS difference in incidence of } \\
\text { new VCF between groups. }\end{array}$ & None & NR \\
\hline
\end{tabular}


Table 1. Continued. Extended.

\begin{tabular}{|c|c|c|c|}
\hline $\begin{array}{c}\text { Cost/Benefit } \\
\text { of Surgical } \\
\text { Intervention }\end{array}$ & $\begin{array}{c}\text { Economic } \\
\text { Considerations }\end{array}$ & $\begin{array}{l}\text { QOL/SF-36 } \\
\text { for CABG/ } \\
\text { THA/TKA }\end{array}$ & Conclusion \\
\hline NR & NR & NR & $\begin{array}{l}\text { Compared with patients who received OMT, } \\
\text { patients who received PV had statistically } \\
\text { significant improvements in QOL for } 36 \mathrm{mo} \text {, } \\
\text { VAS for } 6 \text { mo. }\end{array}$ \\
\hline $\mathrm{CV}$ has a clear advantage. & NR & NR & $\begin{array}{l}\text { SK system is technically superior in } \\
\text { reconstructing the collapse and repair of the } \\
\text { local kyphotic deformity, but this advantage } \\
\text { is not manifest in the main index of } \\
\text { procedure success - namely, pain relief. } \\
\text { Both systems have a high level of safety. }\end{array}$ \\
\hline $\begin{array}{l}\text { Cost/QALY: } \\
\text { BKP cost gain is } \$ 134043 \text {. } \\
\text { But counting for } \\
\text { sensitivity analysis, it } \\
\text { does not show cost- } \\
\text { effectiveness compared } \\
\text { with control. }\end{array}$ & NR & NR & $\begin{array}{l}\text { Study could not demonstrate cost-effectiveness } \\
\text { of BKP over control. }\end{array}$ \\
\hline NR & NR & NR & $\begin{array}{l}\text { Supports KIVA implant as a reliable alternative } \\
\text { technique to BKP for treating fresh }(<3 \\
\text { mo) osteoporotic fractures. } \\
\text { The better radiological reduction of } \\
\text { posttraumatic kyphosis associated with KIVA } \\
\text { may at least theoretically influence the } \\
\text { medium- and long-term results (less back pain, } \\
\text { less frequent adjacent segment fractures). }\end{array}$ \\
\hline NR & NR & NR & $\begin{array}{l}\text { Found no beneficial effects of vertebroplasty } \\
\text { over a sham procedure at } 12 \text { or } 24 \text { mo among } \\
\text { patients with painful osteoporotic vertebral } \\
\text { fractures. }\end{array}$ \\
\hline
\end{tabular}

When patients have no risk factors, conservative treatment for an initial 3 wk will be helpful in the treatment of acute OVCFs.

However, if the patient failed conservative treatment, kyphoplasty also resulted in excellent results at 1 y after trauma.

Both BKP and VP improved disability and pain scores, but BKP offered better spinal deformity correction and resulted in less cement leakage than VP. 
Table 1. Continued.

\begin{tabular}{|c|c|c|c|}
\hline $\begin{array}{l}\text { Author/Year of } \\
\text { Publication }\end{array}$ & Baseline Characteristics & Pain Relief & Disability \\
\hline Movrin $^{38}$ & $\begin{array}{l}\text { 1. Total No. patients: } 107 . \\
\text { 2. BKP ( } 46 \text { patients, average age } \\
67.8 \text { y) versus CT }(61 \text { patients, } \\
\text { average age } 73.8 \text { y) from Jan } \\
2007 \text { to Dec } 2008 \text {. } \\
\text { 3. Follow-up at } 1 \mathrm{~d}, 6 \mathrm{wk}, 12 \mathrm{wk} \text {, } \\
1 \text { y postoperatively. } \\
\text { Level II study. }\end{array}$ & $\begin{array}{l}\text { VAS: } \\
\text { BKP } 2.0 \text { versus CT } 3.8 \text {. } \\
\text { BKP superior to CT (SS). }\end{array}$ & NR \\
\hline Nakano et $\mathrm{al}^{39}$ & $\begin{array}{l}\text { 1. Total No. patients: } 80 . \\
\text { 2. VP ( } 40 \text { patients, average age } 79 \\
\text { y) versus CT ( } 40 \text { patients, } \\
\text { average age } 78 \text { y) from Jun } \\
2002 \text { to Feb } 2007 \text {. } \\
\text { 3. Follow-up: } 6,12,24 \mathrm{mo} . \\
\text { VP } 18.9 \text { mo. } \\
\text { CT } 26.2 \text { mo. } \\
\text { Level II study. }\end{array}$ & $\begin{array}{l}\text { VAS }(\mathrm{cm}) \text { : } \\
\text { VP: } 0.5 \\
\text { CT: } 2.0 \\
\text { Both VP and CT had SS lower } \\
\text { VAS score from baseline, and } \\
\text { VP was superior to CT for pain } \\
\text { reduction at } 24 \text { mo (SS). }\end{array}$ & NR \\
\hline Otten et $\mathrm{al}^{25}$ & $\begin{array}{l}\text { 1. Total No. patients: } 52 \text {. } \\
\text { 2. Kiva VP ( } 26 \text { patients, average } \\
\text { age } 73.6 \text { y) versus BKP }(26 \\
\text { patients, average age } 66.4 \text { y) } \\
\text { 3. Pathological compression } \\
\text { fractures. } \\
\text { 4. Follow-up period of } 6 \text { mo } \\
\text { 5. Matched pairs between Kiva } \\
\text { VP and BKP based on vertebral } \\
\text { body treated, age. } \\
\text { Level II study. }\end{array}$ & $\begin{array}{l}\text { VAS: } \\
\text { Both Kiva VP and BKP lowered } \\
\text { VAS scores. But Kiva has SS } \\
\text { lower VAS score } 6 \text { mo after } \\
\text { treatment than BKP group } \\
(P<.0001) \text {. }\end{array}$ & $\begin{array}{l}\text { ODI: } \\
\text { Both Kiva VP and BKP lowered } \\
\text { ODI scores. No SS difference } \\
\text { between Kiva and BKP. }\end{array}$ \\
\hline Staples et $\mathrm{al}^{40}$ & $\begin{array}{l}\text { 1. Total No. patients: } 78 \text {. } \\
\text { 2. Placebo ( } 40 \text { patients) versus VP } \\
(38 \text { patients) from Apr } 2004 \text { to } \\
\text { Oct } 2010 \text {. } \\
\text { 3. Follow-up: } 1 \mathrm{wk}, 1 \mathrm{mo}, 3 \mathrm{mo}, 6 \\
\text { mo, } 1 \text { y, } 2 \text { y. } \\
\text { Level II study. }\end{array}$ & NR & NR \\
\hline Tutton et $\mathrm{al}^{14}$ & $\begin{array}{l}\text { 1. Total No. patients: } 253 \text {. } \\
\text { 2. KIVA ( } 127 \text { patients, average } \\
\text { age } 76.03 \text { y) versus BKP ( } 126 \\
\text { patients, average age } 75.09 \text { y) } \\
\text { from Aug } 2010 \text { to May } 2013 \text {. } \\
\text { 3. Follow-up: } 12 \text { mo. } \\
\text { Level I study. }\end{array}$ & $\begin{array}{l}\text { VAS: } \\
\text { KIVA: }-70.8 \\
\text { BKP: }-71.8 \\
\text { Both improved VAS score from } \\
\text { baseline (SS), but NSS } \\
\text { compared with each other. }\end{array}$ & $\begin{array}{l}\text { ODI: } \\
\text { KIVA: }-38.1 \\
\text { BKP: }-42.2 \\
\text { Both improved VAS score from } \\
\text { baseline (SS), but NSS } \\
\text { compared with each other. }\end{array}$ \\
\hline Van Meirhaeghe $^{41}$ & $\begin{array}{l}\text { 1. Total No. patients: } 300 \text {. } \\
\text { 2. BKP (149 patients, average age } \\
72.2 \text { y) versus NSM }(151 \\
\text { patients, average age } 74.1 \mathrm{y}) \text {. } \\
\text { 3. Follow-up: } 24 \text { mo. } \\
\text { Level II study. }\end{array}$ & $\begin{array}{l}\text { VAS: } \\
\text { BKP more back pain relief. } \\
\text { BKP } 2.82 \text { versus NSM } 3.65 \text { (SS } \\
\text { BKP superior to NSM). }\end{array}$ & $\begin{array}{l}\text { RMDQ: } \\
\text { Less in BKP than in NSM. } \\
\text { BKP } 8.87 \text { versus NSM } 10.3 \text {. } \\
\text { Mobility: } \\
\text { TUG: } \\
\text { BKP } 13.8 \text { versus NSM } 16.9 \text {. } \\
\text { NSS between groups. }\end{array}$ \\
\hline
\end{tabular}


Table 1. Continued. Extended.

\begin{tabular}{|c|c|c|c|}
\hline Quality of Life & Kyphotic Angle (Grades) & Vertebral Height & Cement Extravasation \\
\hline NR & $\begin{array}{l}\text { Cobb's technique to calculate } \\
\text { segmental kyphotic angle across } \\
\text { fractured level: } \\
\text { BKP } 5.4 \text { versus CT } 10.6 \text {. }\end{array}$ & NR & BKP: $8.7 \%$ \\
\hline NR & NR & $\begin{array}{l}\text { Deformity index: } \\
\text { VP: } 1.61 \\
\text { CT: } 1.37 \\
\text { APHC: } \\
\text { VP: } 70.2 \\
\text { CT: } 49.1\end{array}$ & $\begin{array}{l}\text { VP: } 1 \text { patient } \\
\text { (intervertebral) }\end{array}$ \\
\hline NR & NR & $\begin{array}{l}\text { Both Kiva and BKP showed SS } \\
(P<.001) \text { increase in anterior } \\
\text { and midwall height after } \\
\text { operations, but no SS difference } \\
\text { after } 6 \text { mo compared with post- } \\
\text { op. }\end{array}$ & $\begin{array}{l}6 \text { cases of cement } \\
\text { extravasation in Kiva } \\
\text { and } 8 \text { cases in BKP. } \\
\text { No SS difference } \\
\text { across groups. }\end{array}$ \\
\hline NR & NR & NR & $\begin{array}{l}\text { VP: Cement leakage at } \\
18 \text { of the } 45 \text { treated } \\
\text { levels }(40 \%) .\end{array}$ \\
\hline NR & NR & NR & $\begin{array}{l}\text { Lower in the KIVA } \\
\text { group }(64.6 \%) \text { versus } \\
\text { BKP }(64.5 \%) \text {. }\end{array}$ \\
\hline $\begin{array}{l}\text { EQ-5D: } \\
\text { SS improvement with BKP. } \\
\text { BKP } 0.61 \text { versus NSM } 0.53 \text {. }\end{array}$ & $\begin{array}{l}\text { Index fracture kyphotic } \\
\text { angulation: } \mathrm{SS} \text {, } \\
\text { BKP } 3.13^{\circ} \text { versus NSM } 0.82^{\circ} \text {. }\end{array}$ & $\begin{array}{l}\text { Anterior vertebral height: } \\
\text { BKP improved } 6.7 \% \text { versus NSM } \\
\text { improved } 1.1 \% \text {. } \\
\text { Midline vertebral height: } \\
\text { BKP improved } 5.9 \% \text { versus NSM } \\
\text { worsened } 1.9 \% \text {. } \\
\text { BKP superior (SS) to NSM. }\end{array}$ & $\begin{array}{l}51 \text { bodies }(27.1 \%) \text { with } \\
\text { cement leakage. }\end{array}$ \\
\hline
\end{tabular}


Table 1. Continued. Extended.

\begin{tabular}{|c|c|c|c|}
\hline $\begin{array}{c}\text { Spinal Canal/ } \\
\text { Foramen } \\
\text { Extravasation }\end{array}$ & New VF & Adverse Event & $\begin{array}{l}\text { Optimal } \\
\text { Intervention } \\
\text { Time }\end{array}$ \\
\hline NR & $\begin{array}{l}\text { Adjacent fractures: } \\
\text { BKP: } 3 \\
\text { CT: } 10\end{array}$ & NR & NR \\
\hline VP: 4 patients & $\begin{array}{l}\text { VP: } 5 \\
\text { CT: } 11\end{array}$ & $\begin{array}{l}\text { VP: } 1 \text { patient suffered from } \\
\text { urinary tract infection. } \\
\text { CT: } 6 \text { patients had gastritis } \\
\text { and anorexia, } 2 \text { patients } \\
\text { with leg and facial edema. }\end{array}$ & NR \\
\hline NR & $\begin{array}{l}\text { New fractures in } 3 \text { patients in } \\
\text { Kiva and } 14 \text { patients in BKP. } \\
\text { New fractures occur SS lower in } \\
\text { Kiva }(P<.0001) \text { than in BKP } \\
\text { after } 6 \text { mo. }\end{array}$ & NR & $\begin{array}{l}\text { Mean operation } \\
\text { time per } \\
\text { vertebra was } \\
12.7 \text { min for } \\
\text { Kiva and } 46.5 \\
\text { min for BKP. }\end{array}$ \\
\hline NR & $\begin{array}{l}\text { New adjacent level: } \\
\text { Placebo: } 3 \\
\text { VP: } 6 \\
\text { New nonadjacent: } \\
\text { Placebo: } 7 \\
\text { VP: } 10 \\
\text { New level: } \\
\text { Placebo: } 0 \\
\text { VP: } 1\end{array}$ & NR & NR \\
\hline NR & NR & $\begin{array}{l}\text { Only procedure-related devices. } \\
\text { KIVA: } 3 \text { patients, herpes } \\
\text { zoster, postprocedural pain, } \\
\text { pruritus. } \\
\text { BKP: } 4 \text { patients, airway } \\
\text { complication of anesthesia, } \\
\text { back pain, ischemic stroke, } \\
\text { rash. }\end{array}$ & NR \\
\hline NR & $\begin{array}{l}\text { BKP: } 11 \text { patients versus NSM } 7 \\
\text { patients. }\end{array}$ & $\begin{array}{l}\text { All AEs occurred in the first } \\
30 \text { d. } \\
\text { Back pain: } \\
\text { BKP } 20 \text { patients versus NSM } \\
11 \text { patients. } \\
\text { UTI: } \\
\text { BKP } 10 \text { patients versus NSM } \\
3 \text { patients. } \\
\text { Nausea/vomiting: } \\
\text { BKP } 12 \text { patients versus NSM } \\
4 \text { patients, } \\
\text { Hematomas: } \\
\text { BKP } 4 \text { patients. }\end{array}$ & NR \\
\hline
\end{tabular}


Table 1. Continued. Extended.

\begin{tabular}{|c|c|c|c|}
\hline $\begin{array}{c}\text { Cost/Benefit } \\
\text { of Surgical } \\
\text { Intervention } \\
\end{array}$ & $\begin{array}{c}\text { Economic } \\
\text { Considerations } \\
\end{array}$ & $\begin{array}{l}\text { QOL/SF-36 } \\
\text { for CABG/ } \\
\text { THA/TKA }\end{array}$ & Conclusion \\
\hline NR & NR & NR & $\begin{array}{l}\text { Correction of the vertebral morphology and } \\
\text { prevention of further deterioration achieved } \\
\text { with BKP probably has a positive effect on } \\
\text { the spinal biomechanics and thus reduces the } \\
\text { incidence of subsequent fracture. }\end{array}$ \\
\hline NR & NR & NR & $\begin{array}{l}\text { Vertebroplasty using CPC following cavity } \\
\text { formation of VB provided better clinical and } \\
\text { radiological results than conservative } \\
\text { treatment for osteoporotic burst fracture in } \\
\text { patients without neural deficit. }\end{array}$ \\
\hline NR & NR & NR & $\begin{array}{l}\text { Kiva is superior to BKP with pain VAS scores. } \\
\text { Disability improvement was similar across } \\
\text { Kiva and BKP. Both groups had vertebral } \\
\text { height restoration and same risk of cement } \\
\text { extravasation. Kiva operation time is shorter. }\end{array}$ \\
\hline
\end{tabular}
provide superior pain relief or functional benefit compared with placebo, but the study did not observe an increase in subsequent fracture risk beyond that experienced by those with vertebral fractures. pain, decrease disability, and improve quality of life in patients with painful VCFs.

The Kiva system is noninferior to BKP in its ability to safely relieve pain and improve function in the treatment of osteoporotic VCFs.

Compared with NSM, BKP rapidly reduces pain and improves function, disability, and QOL during the course of $2 \mathrm{y}$ and the reduction in pain. EQ-5D QOL, patient satisfaction, and kyphotic angulation remain statistically significant at all time points. 
Table 1. Continued.

\begin{tabular}{|c|c|c|c|}
\hline $\begin{array}{l}\text { Author/Year of } \\
\text { Publication }\end{array}$ & Baseline Characteristics & Pain Relief & Disability \\
\hline Wang et $\mathrm{al}^{42}$ & $\begin{array}{l}\text { 1. Total No. patients: } 107 . \\
\text { 2. HVCV ( } 50 \text { patients, average } \\
\text { age } 69.43 \text { y) versus BKP ( } 51 \\
\text { patients, average age } 68.63 \text { y) } \\
\text { from Jan } 2012 \text { to Feb } 2014 \text {. } \\
\text { 3. Preoperatively, postoperatively, } \\
3 \text { mo and } 1 \text { y follow-up. } \\
\text { Level I study. }\end{array}$ & $\begin{array}{l}\text { VAS: } \\
\text { NSS between groups } \\
\text { preoperatively, postoperatively, } \\
\text { at } 3 \text { mo and at } 1 \text { y follow-up. }\end{array}$ & $\begin{array}{l}\text { ODI: } \\
\text { NSS between groups } \\
\text { preoperatively, at } 3 \text { mo and at } 1 \\
\text { y follow-up. }\end{array}$ \\
\hline Werner et al1 $1^{5}$ & $\begin{array}{l}\text { 1. Total No. patients: } 65 \\
\text { 2. BKP ( } 32 \text { patients, average age } \\
66 \text { y) versus VBS ( } 33 \text { patients, } \\
\text { average age } 73 \text { y). } \\
\text { 3. Follow-up: NR. } \\
\text { Level I study. }\end{array}$ & NR & NR \\
\hline Yang et $\mathrm{al}^{43}$ & $\begin{array}{l}\text { 1. Total No. patients: } 221 \text {. } \\
\text { 2. VP (109 patients, average age } \\
73.39 \text { y) versus BKP }(112 \\
\text { patients, average age } 73.48 \text { ) } \\
\text { from Jan } 2008 \text { to Oct } 2012 \text {. } \\
\text { 3. Follow-up: NR. } \\
\text { Level I study. }\end{array}$ & NR & NR \\
\hline Yokohama et $\mathrm{al}^{44}$ & $\begin{array}{l}\text { 1. Total No. patients: } 66 \text {. } \\
\text { 2. VP ( } 28 \text { patients, average age } 74 \\
\text { y) versus BKP ( } 38 \text { patients, } \\
\text { average age } 75.5 \text { y) from Jan } \\
2008 \text { to Apr } 2012 \text {. } \\
\text { 3. Follow-up: NR. } \\
\text { Level II study. }\end{array}$ & $\begin{array}{l}\text { VAS: } \\
\text { VP } 1.59 \\
\text { BKP } 2.39 \\
\text { NSS }\end{array}$ & NR \\
\hline
\end{tabular}

Abbreviations: AE, adverse event; AP, anterior-posterior; APHC, anteroposterior height comparison; BF, bone filler; BKP, balloon kyphoplasty; CABG, coronary artery bypass grafting; CPC, calcium phosphate cement; CT, conservative therapy; CV, confidence vertbroplasty; EQ-5D, EuroQol five dimensions; HVCV, high viscosity cement vetebroplasty; LBP, low-back pain; NR, not relevant; NRS, numerical rating scale; NSM, nonsurgical management; NSS, not statistically significant; ODI, Oswestry Disability Index; OMT, optimal medical therapy; OVCF, osteoporotic vertebral compression fracture; PCS, physical component summary; PVP, percutaneous vertebroplasty; QALY, quality-adjusted life year; QOL, quality of life; QUALEFFO, Quality of Life Questionnaire of the European Foundation for Ostoporosis; RMDQ, Roland-Morris Disability Questionnaire; SF-36, 36-Item Short Form Health Survey; SI, sagital index; SK, sky kyphoplasty; SKP, shield kyphoplasty; SS, statistically significant; THA, total hip arthroplasty; TKA, total knee arthroplasty; TUG, time up-and-go; UTI, urinary tract infection; VAS, visual analog scale; VBH, vertebral body height; VBS, vertebral body stenting; VCF, vertebral compression fracture; VP, vertebroplasty.

the meta-analysis. Table 2 summarizes the treatment comparison results across pooled studies, showing standardized mean differences of change from baseline for each treatment group and $P$ values for treatment comparisons.

Pain was reported through multiple metrics, including 10-point back pain scores, SF-36 bodily pain score, and VAS score (Table 1), but there was a sufficient number of studies to only compare VAS scores (Table 2). BKP showed some tendency for slightly higher change in VAS scores from baseline than VP and VAI for the majority of studies with an overall response ratio of $1.13(0.45,2.83$; Figure 1$)$ and $1.04(0.26,4.20$; Figure 2$)$, respectively. In terms of pain reduction from baseline, BKP $(-4.05[-4.81$, $-3.29])$ was not significantly different than VP $(-3.88[-4.74,-3.02], P=.774)$ but showed significantly greater improvement compared with NSM $(-2.66[-3.75,-1.56], P=.041$; Figure 3$)$ and VAI $(-2.77[-3.51,-2.04], P=.018)$. The difference between VP and NSM in terms of pain reduction was not significant $(P=.085)$. There was substantial heterogeneity in the BKP $\left(\mathrm{I}^{2}=95\right)$, VP $\left(\mathrm{I}^{2}=86\right)$, VAI $\left(\mathrm{I}^{2}=84\right)$, and NSM $\left(\mathrm{I}^{2}=96\right)$ arms.

Disability was reported through RMDQ score, disability days, ODI, and TUG metrics (Table 1), but there was a sufficient number of studies to only compare ODI and RMDQ (Table 2). The response 
Table 1. Continued. Extended.

\begin{tabular}{|c|c|c|c|c|}
\hline & Quality of Life & Kyphotic Angle (Grades) & Vertebral Height & Cement Extravasation \\
\hline NR & & NR & $\begin{array}{l}\text { Lower anterior height restoration } \\
\text { rate in HVCV (30.04) versus } \\
\text { BKP ( } 42.65), \text { SS. } \\
\text { At } 1 \text { y follow-up, NSS loss of } \\
\text { height in both groups. }\end{array}$ & $\begin{array}{l}\text { Lower in HVCV ( } 9 \text { of } \\
68) \text { versus BKP } \\
\text { (22 of } 72 \text { ). }\end{array}$ \\
\hline NR & & $\begin{array}{l}\text { BKP: } 4.5^{\circ} \\
\text { VBS: } 4.7^{\circ} \\
\text { NSS }\end{array}$ & NR & $\begin{array}{l}\text { Major cement leakage } \\
\quad \text { (No. patients): } \\
\text { BKP: } 4 \\
\text { VBS: } 5 \\
\text { NSS }\end{array}$ \\
\hline NR & & $\begin{array}{l}\text { BKP: } 7.82^{\circ} \\
\text { VP: } 3.82^{\circ} \\
\text { BKP superior to VP (SS). }\end{array}$ & $\begin{array}{l}\text { Preadjusted Postoperative: } \\
\text { VP: } 23.48 \\
\text { BKP: } 21.42 \\
\text { Adjusted Postoperative: } \\
\text { VP: } 21.988 \\
\text { BKP: } 22.869 \\
\text { BKP superior to VP (SS). }\end{array}$ & $\begin{array}{l}\text { VP: } 56 \text { cases } \\
\text { BKP: } 49 \text { cases } \\
\text { NSS }\end{array}$ \\
\hline NR & & $\begin{array}{l}\text { Kyphotic change: } \\
\text { VP: } 7.0 \\
\text { BKP: } 6.9 \\
\text { NSS }\end{array}$ & $\begin{array}{l}\text { Anterior: } \\
\text { VP: } 3.6 \\
\text { BKP: } 3.6 \\
\text { NSS } \\
\text { Central: } \\
\text { VP: } 2.5 \\
\text { BKP: } 2.0 \\
\text { NSS } \\
\text { Posterior: } \\
\text { VP: } 0.9 \\
\text { BKP: } 0.5 \\
\text { NSS }\end{array}$ & $\begin{array}{l}\text { Cement leak: } \\
\text { VP: } 16 \text { vertebral bodies } \\
\text { BKP: } 9 \text { vertebral bodies } \\
\text { SS }\end{array}$ \\
\hline
\end{tabular}

ratio plot of RMDQ scores showed NSM had some tendency for slightly higher changes from baseline compared with VP with a response ratio of 1.13 $(0.13,9.53$; Figure 4). The overall mean effect of RMDQ for the NSM group was $-2.86(-4.71$, $-1.01)$ and for the VP group was $-2.41(-4.68$, $-0.15)$ with no significant difference $(P=.763$; Figure 5). A response ratio plot of ODI scores showed that BKP treatment groups had some tendency to have slightly higher changes from baseline compared with VP groups with a response ratio of $1.02(0.18,5.76$; Figure 6$)$ and compared with VAI with a response ratio of $1.51(0.27,8.58$; Figure 7). For disability index (ODI) reduction, BKP $(-4.20[-5.35,-3.05])$ showed slightly less improvement versus VP (-6.61 [-9.59, -3.63], $P=.140$; Figure 8$)$. The reduction in ODI was significantly higher for BKP than VAI $(-1.91[-2.22$, $-1.61], P<.001)$. There was substantial heteroge- neity in the BKP $\left(\mathrm{I}^{2}=96\right)$ and $\mathrm{VP}\left(\mathrm{I}^{2}=97\right)$ arms but less observed heterogeneity in the VAI group $\left(\mathrm{I}^{2}=47\right)$.

Quality of life (QOL) was reported through SF36 PCS, EQ-5D score, and generally through the SF-36 metric. Due to the limited number of studies, overall mean effects were not calculated for BKP and VP. For quality of life (EQ-5D), NSM showed a mean improvement of $1.41(-0.29,3.11)$. There was substantial heterogeneity in the NSM $\left(\mathrm{I}^{2}=98\right)$ arm.

A response ratio plot comparing $\mathrm{BKP}$ with $\mathrm{VP}$ for kyphotic angular correction (KAC) (Figure 9) showed high variability across studies, with an overall mean estimate of $1.46(0.84,2.51)$ for response ratios. A forest plot of standardized mean differences from baseline for $\mathrm{KAC}$ is shown in Figure 10. There were not enough studies to complete an analysis of the NSM or VAI group. For KAC, BKP $(-1.17$ [-1.45, -0.89$])$ showed more 
Table 1. Continued. Extended.

\begin{tabular}{|c|c|c|c|}
\hline $\begin{array}{l}\text { Spinal Canal/ } \\
\text { Foramen } \\
\text { Extravasation }\end{array}$ & New VF & Adverse Event & $\begin{array}{l}\text { Optimal } \\
\text { Intervention } \\
\text { Time }\end{array}$ \\
\hline NR & $\begin{array}{l}1 \mathrm{HVCV} \text { patient versus } 4 \text { BKP } \\
\text { patients. } \\
\text { Subsequent fractures NSS. }\end{array}$ & $\begin{array}{l}\text { BKP group: } \\
1 \text { patient experienced severe } \\
\text { discogenic back pain related } \\
\text { to a disc leak; } \\
1 \text { patient asymptomatic cement } \\
\text { emboli in the right lung } \\
\text { related to venous leakage. }\end{array}$ & NR \\
\hline NR & NR & $\begin{array}{l}\text { Material-related complication: } \\
\text { Cannula: } 5 \text { VBS } \\
\text { Balloon: 1 BKP, 1 VBS } \\
\text { Stent: } 3 \text { VBS }\end{array}$ & NR \\
\hline NR & NR & NR & NR \\
\hline NR & NR & NR & NR \\
\hline
\end{tabular}

kyphotic angle reduction than VP $(-1.07[-1.42$, $-0.72] ; P=.658)$, although the difference is not significant. There was substantial heterogeneity in the BKP $\left(\mathrm{I}^{2}=82\right)$ and VP $\left(\mathrm{I}^{2}=83\right)$ arms.

For anterior and posterior vertebral height restoration, BKP showed higher positive change in response compared with VP. Mean estimates for response ratios for anterior and posterior are 1.40 (0.94, 2.07; Figure 11) and 1.62 (1.23, 2.12; Figure 12), respectively, for BKP over VP. Forest plots of standardized mean differences from baseline for vertebral heights are shown in Figures 13 14. Only BKP and VP were compared due to the number of eligible studies.

For anterior vertebral height change in response, both treatments showed improvement by increasing restoration. BKP had a mean response change of $1.23(0.87,1.58)$, which was not significantly higher than VP at $0.98(0.78,1.17 ; P=.226)$. There was substantial heterogeneity in the BKP $\left(\mathrm{I}^{2}=81\right)$ arm but not the VP $\left(\mathrm{I}^{2}=20\right)$ arm.

For midline vertebral height change in response, BKP was $1.12(0.87,1.38)$. There were not enough studies for VP or VAI. There was midheterogeneity in the BKP $\left(\mathrm{I}^{2}=37\right)$ arm.

For posterior vertebral height restoration, BKP $(0.54[0.31,0.77])$ had slightly higher change than VP $(0.38[0.18,0.58])$, but the difference was not significant $(P=.293)$. There was moderate and minimal heterogeneity in the BKP $\left(\mathrm{I}^{2}=60\right)$ and VP $\left(I^{2}=0\right)$ arms, respectively.

The percentages of patients with subsequent fractures were not significantly different for BKP (32.7\%; 95\% confidence interval [CI] 8.8\%, 56.6\%) and VP $(28.3 \% ; 95 \%$ CI $7.0 \%, 49.7 \% ; P=.790)$ or for BKP and NSM $(15.9 \%$; 95\% CI 5.2\%, 26.6\%; $P=.207$; Figure 15). There was also no significant difference between VP and NSM $(P=.307)$. There 
Table 1. Continued. Extended.

\begin{tabular}{|c|c|c|c|}
\hline $\begin{array}{c}\text { Cost/Benefit } \\
\text { of Surgical } \\
\text { Intervention }\end{array}$ & $\begin{array}{c}\text { Economic } \\
\text { Considerations }\end{array}$ & $\begin{array}{l}\text { QOL/SF-36 } \\
\text { for CABG/ } \\
\text { THA/TKA }\end{array}$ & Conclusion \\
\hline NR & NR & NR & $\begin{array}{l}\text { HVCV has a lower cement leakage rate. } \\
\text { HVCV is recommended for the treatment of } \\
\text { OVCFs. } \\
\text { BKP is more effective in vertebral height } \\
\text { restoration. }\end{array}$ \\
\hline NR & $\begin{array}{l}\text { BKP: } \\
1 \text { level: } \$ 5300 \\
2 \text { levels: } \$ 5700 \\
3 \text { levels: } \$ 6300 \\
\text { VBS: } \\
1 \text { level: } \$ 3750 \\
2 \text { levels: } \$ 6950 \\
3 \text { levels: } \$ 10400\end{array}$ & NR & $\begin{array}{l}\text { No beneficial effect of vertebral body stenting } \\
\text { over balloon kyphoplasty with regard to } \\
\text { kyphotic correction, cement leakage. }\end{array}$ \\
\hline NR & NR & NR & $\begin{array}{l}\text { Vertebroplasty-BF: } \\
\text { Bone cement distribution was more } \\
\text { physiological. } \\
\text { Lumped distribution was avoided. } \\
\text { Sufficient bone cement injection was possible } \\
\text { without increasing the rate of bone cement } \\
\text { leakage. } \\
\text { It can be considered as a compatible option for } \\
\text { the osteoporotic compression fracture and } \\
\text { has the advantages of both conventional } \\
\text { vertebroplasty and kyphoplasty. }\end{array}$ \\
\hline NR & NR & NR & $\begin{array}{l}\text { The vertebral height restoration and kyphotic } \\
\text { changes that were achieved after surgery } \\
\text { largely depended on the preoperative } \\
\text { vertebral mobility in not only the patients } \\
\text { treated by VP but also those treated by BKP. } \\
\text { The use of the balloon in BKP contributed little } \\
\text { to the resolution of the vertebral deformities } \\
\text { following surgery. }\end{array}$ \\
\hline
\end{tabular}

was substantial heterogeneity in the BKP $\left(\mathrm{I}^{2}=95\right)$, $\operatorname{VP}\left(\mathrm{I}^{2}=96\right)$ and NSM $\left(\mathrm{I}^{2}=81\right)$ arms.

\section{DISCUSSION}

The drive to produce and collect data in recent years has been enhanced by the controversy initiated by the 2009 sham trials (Buchbinder et al ${ }^{19}$, Kallmes et $\mathrm{al}^{20}$ ) published in NEJM. Approximately 250 manuscripts dedicated to vertebral augmentation have been published annually. Much of the output attempts to further define the safety and efficacy of these procedures and investigate differences between

Table 2. Treatment comparison results across pooled studies indicating standardized mean differences of change from baseline and respective $P$ value. (Note: $\mathrm{n} / \mathrm{a}$ indicates insufficient number of studies for analysis.)

\begin{tabular}{|c|c|c|c|c|c|c|c|c|}
\hline \multirow[b]{2}{*}{ Outcome } & \multicolumn{4}{|c|}{ Mean Change from Baseline } & \multicolumn{4}{|c|}{$P$ Values } \\
\hline & BKP & VP & VAI & NSM & BKP-VP & BKP-NSM & VP-NSM & BKP-VAI \\
\hline Pain VAS score & -4.05 & -3.88 & -2.77 & -2.66 & .774 & .041 & .085 & .018 \\
\hline Oswestry Disability Index & -4.20 & -6.61 & -1.91 & $\mathrm{n} / \mathrm{a}$ & .140 & & & $<.001$ \\
\hline Roland Morris Disability Questionnaire & $\mathrm{n} / \mathrm{a}$ & -2.41 & $\mathrm{n} / \mathrm{a}$ & -2.86 & & & .763 & \\
\hline Kyphotic angular correction & -1.17 & -1.07 & $\mathrm{n} / \mathrm{a}$ & $\mathrm{n} / \mathrm{a}$ & .658 & & & \\
\hline Anterior vertebral height restoration & 1.23 & 0.98 & $\mathrm{n} / \mathrm{a}$ & $\mathrm{n} / \mathrm{a}$ & .226 & & & \\
\hline Posterior vertebral height restoration & 0.54 & 0.38 & $\mathrm{n} / \mathrm{a}$ & $\mathrm{n} / \mathrm{a}$ & .293 & & & \\
\hline Subsequent fracture rate & 0.33 & 0.28 & $\mathrm{n} / \mathrm{a}$ & 0.16 & .790 & .207 & .307 & \\
\hline
\end{tabular}

Abbreviations: BKP, balloon kyphoplasty; NSM, nonsurgical management; VAI, vertebral augmentation with implants; VAS, visual analog scale; VP, vertebroplasty. 


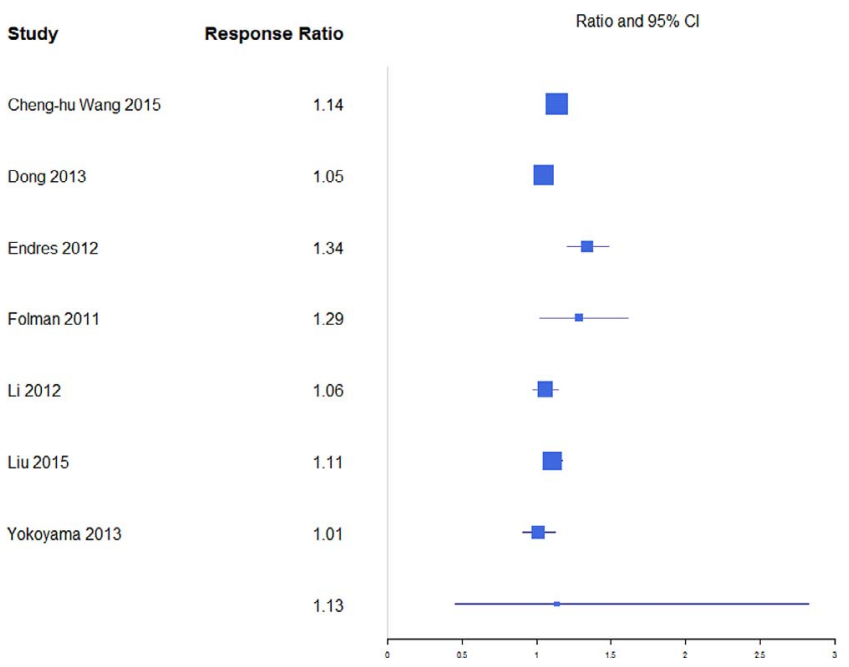

Figure 1. Response ratios of standardized mean differences for visual analog scale pain scores comparing balloon kyphoplasty with vertebroplasty treatment.

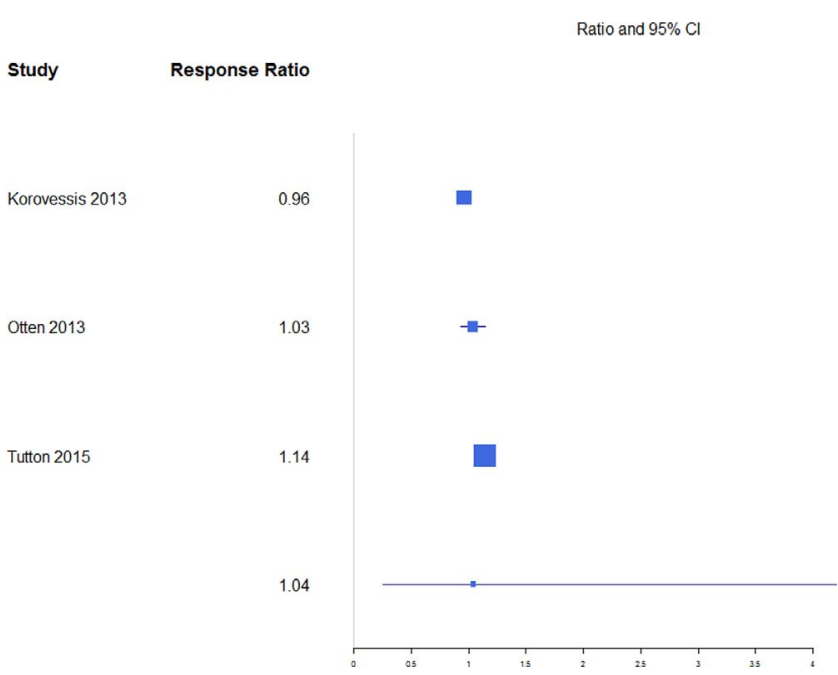

Figure 2. Response ratios of standardized mean differences for visual analog scale pain scores comparing balloon kyphoplasty with vertebral augmentation with implants treatment.
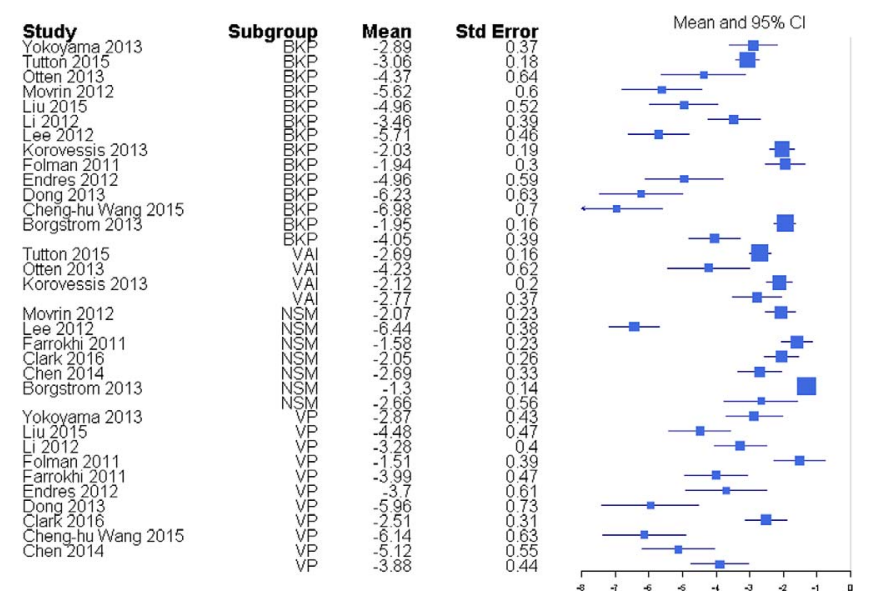

Figure 3. Standardized mean differences of change from faseline for visual analog scale pain scores. Abbreviations: BKP, balloon kyphoplasty; VAI, vertebral augmentation with implants; NSM, nonsurgical management; VP, vertebroplasty.

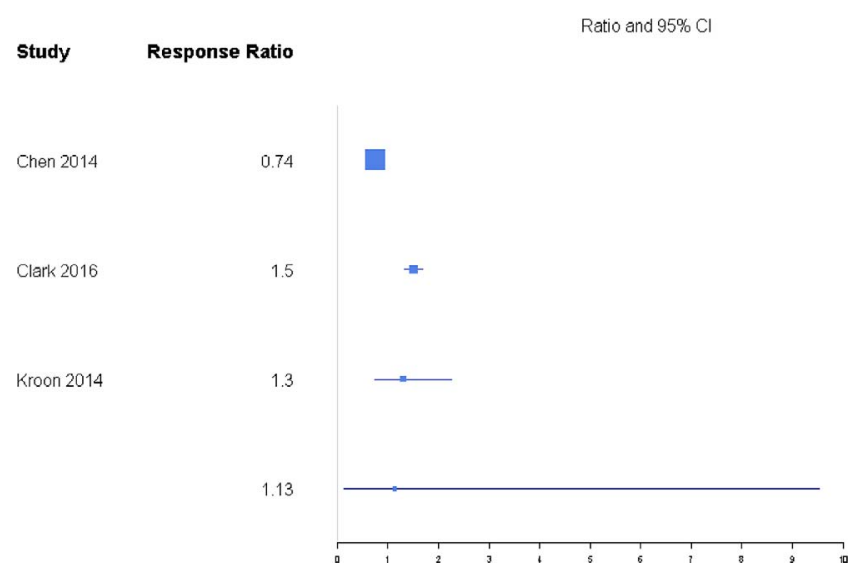

Figure 4. Response ratios of standardized mean differences for Roland Morris Disability Questionnaire comparing nonsurgical management with vertebroplasty treatment.

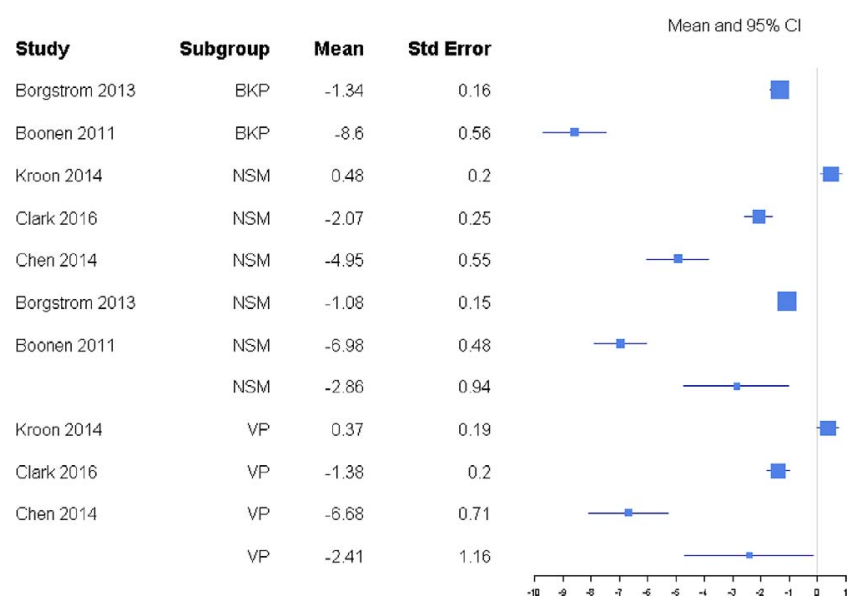

Figure 5. Standardized mean differences of change from baseline for Roland Morris Disability Questionnaire. Abbreviations: BKP, balloon kyphoplasty; NSM, nonsurgical management; VP, vertebroplasty.

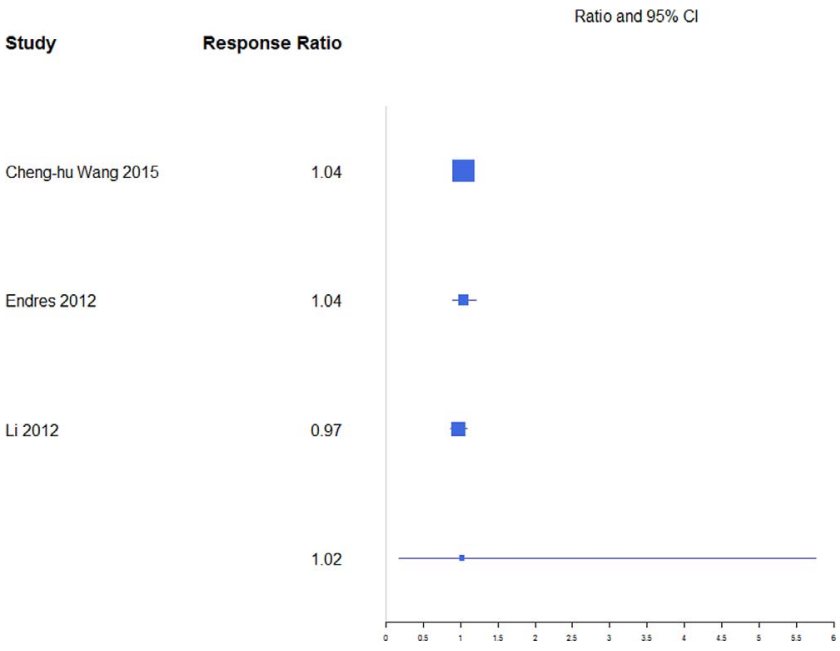

Figure 6. Response ratios of standardized mean differences for Oswestry Disability Index comparing balloon kyphoplasty with vertebroplasty treatment. 
Beall et al.

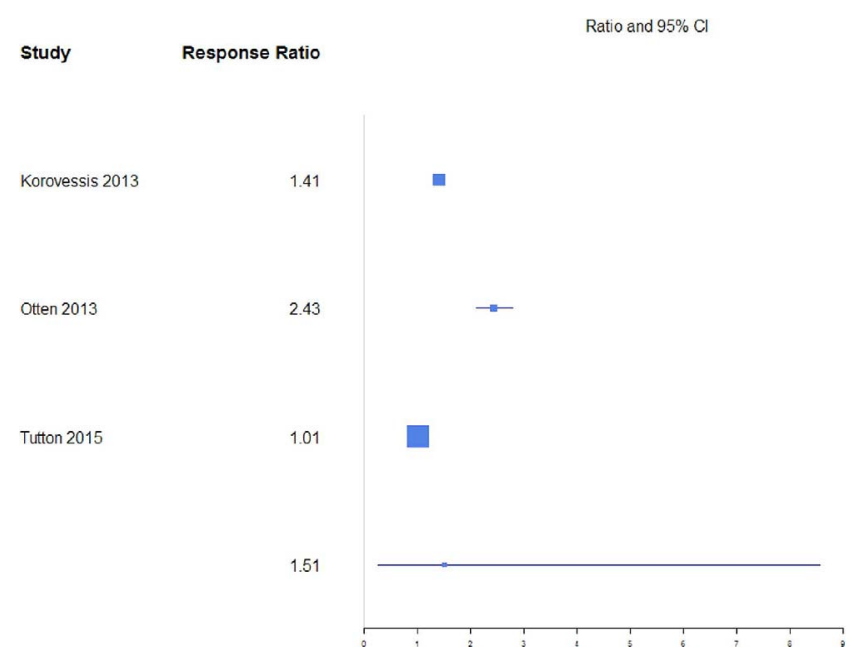

Figure 7. Response ratios of standardized mean differences for Oswestry Disability Index comparing balloon kyphoplasty with vertebral augmentation with implants treatment.

$\begin{array}{lrr}\text { Study } & \text { Subgroup } & \text { Mean } \\ \text { Cheng-hu Wang 2015 } & \text { BKP } & -6.13 \\ \text { Endres 2012 } & \text { BKP } & -2.09 \\ \text { Korovessis 2013 } & \text { BKP } & -2.39 \\ \text { Lee 2012 } & \text { BKP } & -11.09 \\ \text { Li 2012 } & \text { BKP } & -2.04 \\ \text { Otten 2013 } & \text { BKP } & -6.15 \\ \text { Tuton 2015 } & \text { BKP } & -1.94 \\ & \text { BKP } & -4.2 \\ \text { Chen 2014 } & \text { NSM } & -6.59 \\ \text { Lee 2012 } & \text { NSM } & -9.03 \\ \text { Korovessis 2013 } & \text { VAl } & -1.7 \\ \text { Otten 2013 } & \text { VAl } & -2.53 \\ \text { Tutton 2015 } & \text { VAl } & -1.92 \\ & \text { VAl } & -1.91 \\ \text { Chen 2014 } & \text { VP } & -23.44 \\ \text { Cheng-hu Wang 2015 } & \text { VP } & -5.88 \\ \text { Endres 2012 } & \text { VP } & -2.01 \\ \text { Li 2012 } & \text { VP } & -2.11 \\ & \text { VP } & -6.61\end{array}$

Std Erro
0.62
0.29
0.21
0.87
0.26
0.87
0.13
0.59
0.73
0.53
0.17
0.4
0.13
0.16
2.45
0.6
0.38
0.28
1.52

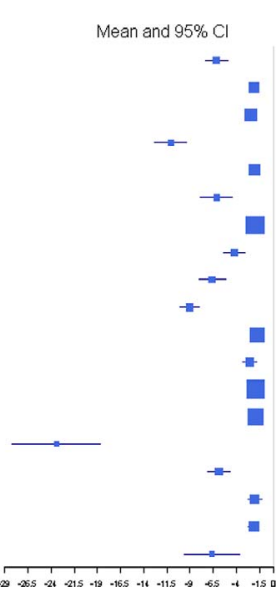

Figure 8. Standardized mean differences of change from baseline for Oswestry Disability Index. Abbreviations: BKP, balloon kyphoplasty; VAI, vertebral augmentation with implants; VP, vertebroplasty.

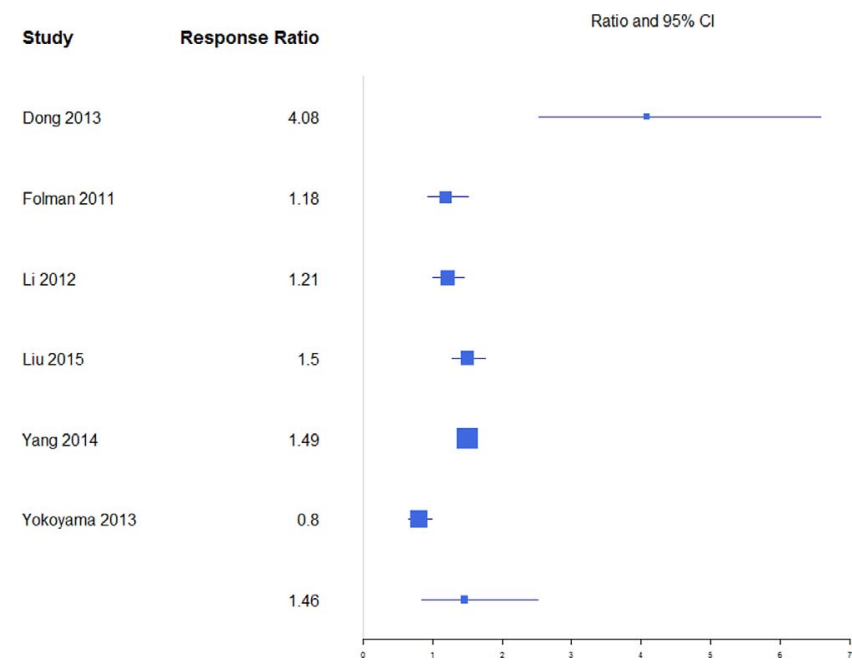

Figure 9. Response ratios of standardized mean differences for kyphotic angular correction comparing balloon kyphoplasty with vetebroplasty treatment.

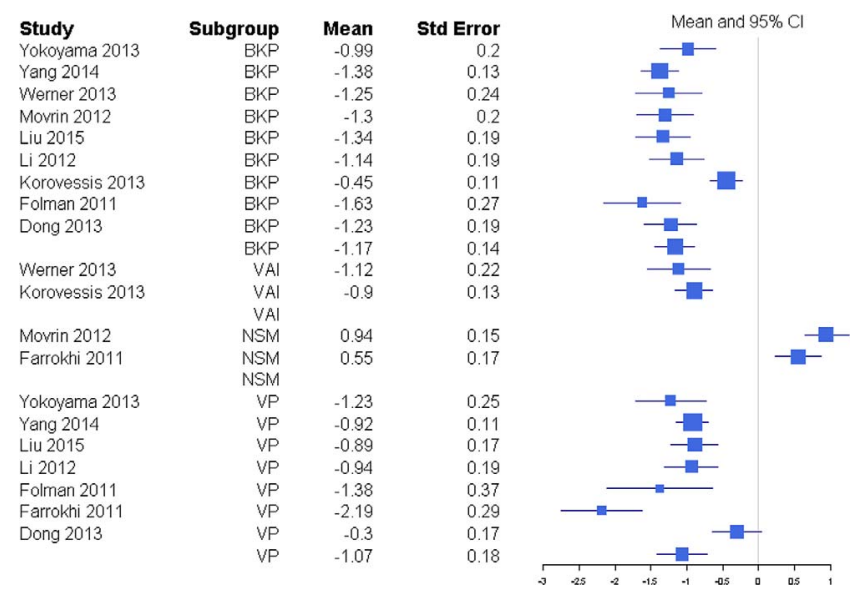

Figure 10. Standardized mean differences of change from baseline for kyphotic angular correction. Negative values indicate reduction in kyphosis angle. Abbreviations: BKP, balloon kyphoplasty; VAI, vertebral augmentation with implants; NSM, nonsurgical management; VP, vertebroplasty.

Study Response Ratio Ratio and $95 \% \mathrm{Cl}$

Dong 2013

Li 2012

Yang 2014

Yokoyama 2013

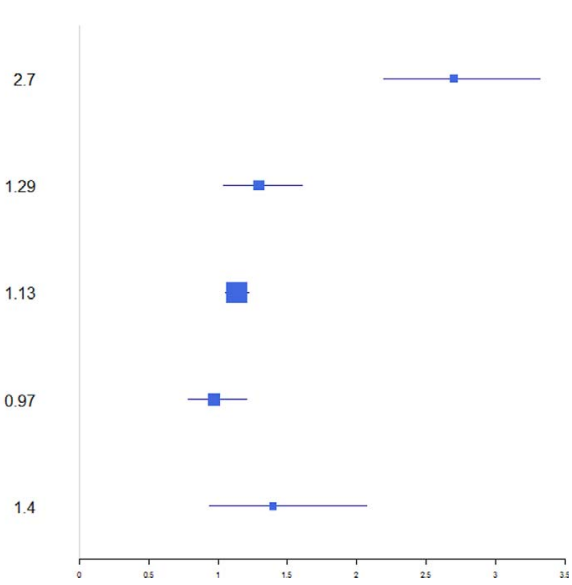

Figure 11. Response ratios of standardized mean differences for anterior vertebral height comparing balloon kyphoplasty with vertebroplasty treatment.

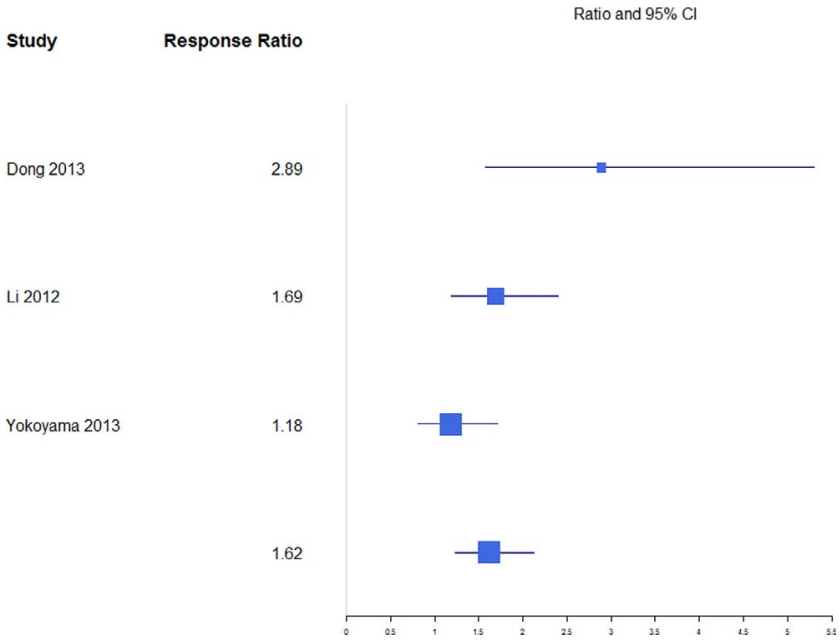

Figure 12. Response ratios of standardized mean differences for posterior vertebral height comparing balloon kyphoplasty with vetebroplasty treatment. 


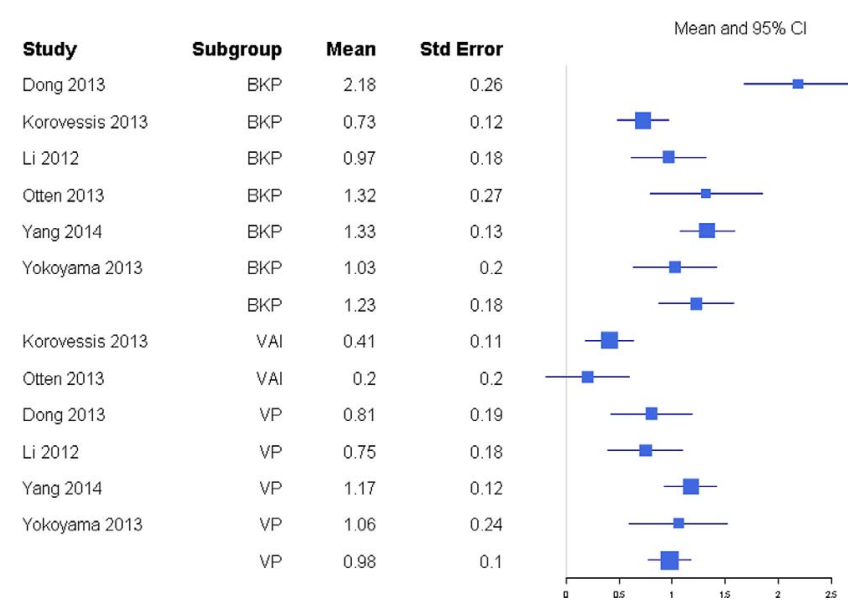

Figure 13. Standardized mean differences of change from baseline for anterior vertebral height. Abbreviations: BKP, balloon kyphoplasty; VAI, vertebral augmentation with implants; VP, vertebroplasty.

the clinical experience with these procedures and the results of the sham trials.

Due to the aforementioned sham trials, there has been a significant decline in the number of vertebral augmentation procedures performed, ${ }^{46-49}$ and various health technology assessments (HTAs) have used this information to recommend against vertebral augmentation in other countries.

These HTA recommendations are at direct odds with medical societies whose recommendations support vertebral augmentation, including a consensus statement ${ }^{50}$ from 8 major medical societies that vertebral augmentation remains a proven, medically appropriate therapy for treatment of painful VCFs refractory to nonoperative medical therapy when performed for medical indications outlined in published standards.

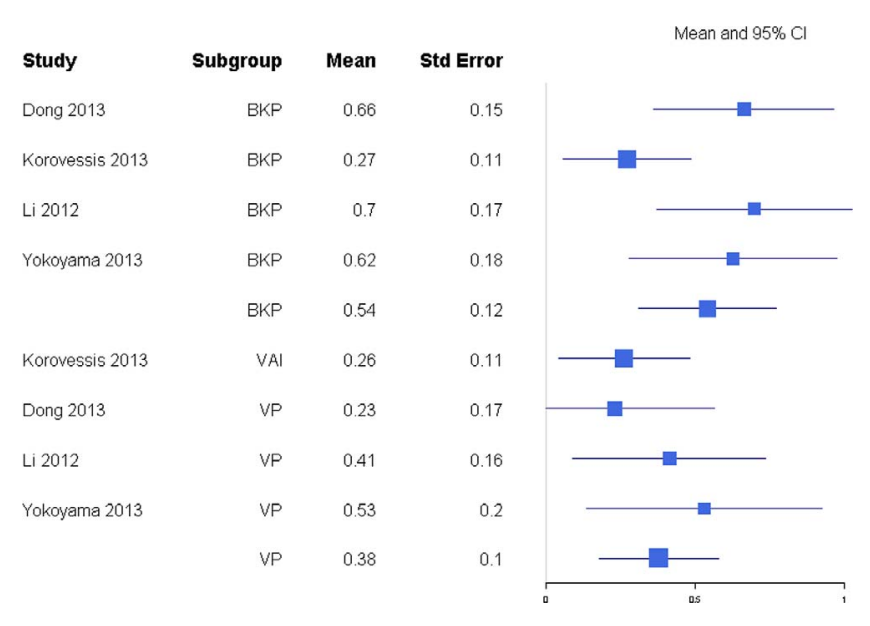

Figure 14. Standardized mean differences of change from baseline for posterior vertebral height. Abbreviations: BKP, balloon kyphoplasty; VAI, vertebral augmentation with implants; VP, vertebroplasty.

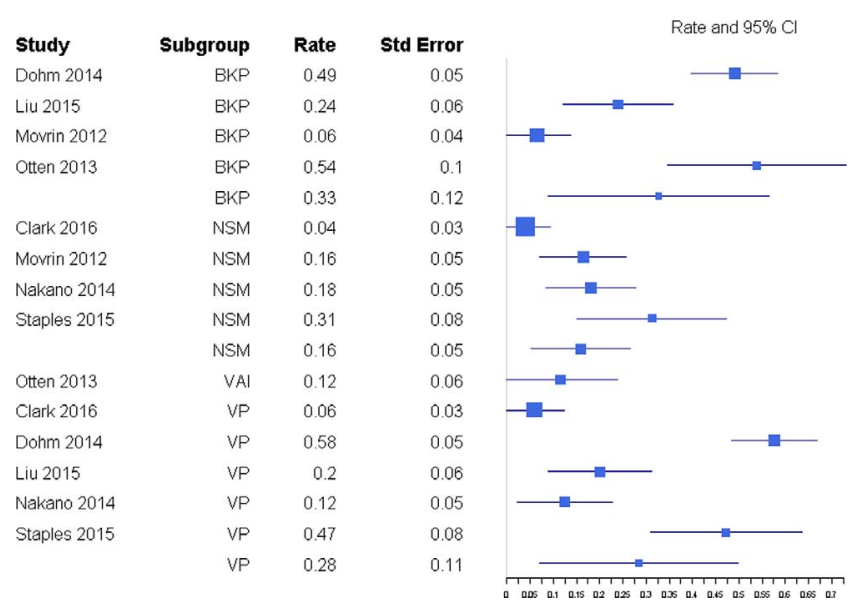

Figure 15. Rate of subsequent fractures. Abbreviations: BKP, balloon kyphoplasty; NSM, nonsurgical management; VAI, vertebral augmentation with implants; VP, vertebroplasty.

An outlier in society recommendations was issued in 2010 by the American Academy of Orthopaedic Surgeons (AAOS) ${ }^{51}$ This was based on an algorithm using levels of evidence to perform hierarchal qualification of data. At the time the AAOS guidelines were issued, far less data were available, and the existing level of evidence hierarchy placed the Buchbinder et $\mathrm{al}^{19}$ and Kallmes et $\mathrm{al}^{20}$ studies as the highest level of evidence. As a consequence, the AAOS guidelines provided a weak recommendation for kyphoplasty and a strong recommendation against VP.

After the NEJM articles, HTA recommendations, and AAOS guidelines, Papanastassiou et $\mathrm{al}^{52}$ conducted a meta-analysis on the effects of kyphoplasty, VP, and NSM to treat VCFs that reported on 27 Level I and Level II studies. Papanastassiou et $\mathrm{al}^{52}$ concluded that BKP and VP provided better pain relief, resulted in fewer additional VCFs than NSM, and concluded that BKP is favored over VP for QOL improvements and cement extravasation. The authors noted that 2 RCTs in their analysis showed no difference between VP and a sham procedure and pointed to significant data heterogeneity recommending further trials to delineate some confounding variables. Since then, there have been several publications, including double-blind RCTs, other Level I studies on vertebral augmentation, and 2 additional VP versus sham studies, that have added to the literature on vertebral augmenation.

A 2016 sham study trial by Clark et $\mathrm{al}^{21}$ compared VP to sham in patients with acute vertebral compression fractures and concluded that VP significantly reduces pain, improves disability 
scores, and achieves greater vertebral height restoration. The findings support use of VP over sham surgery in patients with acute VCFs, which differs from the equivocal result of previous sham trials ${ }^{19,20}$ that concluded no significant difference in pain, function, and QOL between sham patients and patients undergoing VP. A second RCT comparing VP to sham, published in 2016 by Hansen et al, ${ }^{22}$ concluded that there was a statistically signficant VAS score reduction in the VP group as compared with the sham group during their trial period. An explanation for differences in the conclusions is found in one notable publication since the Papanastassiou et $\mathrm{al}^{52}$ study. Anderson et $\mathrm{al}^{53}$ conducted a meta-analysis in 2013 comparing vertebral augmentation with NSM and analyzed the study quality of the 2009 sham trials ${ }^{19,20}$ with the levels of evidence of primary research as adopted by the North American Spine Society. ${ }^{53,54}$ Anderson et al ${ }^{53}$ found the 2009 sham trials should have been classified as providing Level II evidence instead of Level I, based on inclusion and exclusion criteria and the Kallmes et al $^{20}$ trial's unacceptably high crossover rate. Both trials were therefore downgraded to Level II evidence based on objective measures of quality for primary research. ${ }^{53}$

Although the most recent highest-quality literature supports vertebral augmentation over NSM to treat painful VCFs, many HTAs and several national treatment guidelines continue to make outdated, inconsistent recommendations regarding vertebral augmentation. For example, a recent (2015) HTA publication by the Cochrane Collaboration recommended against using $\mathrm{VP}$ to treat VCFs in routine clinical practice. ${ }^{55}$ This recommendation disregarded 11 RCTs and 1 "quasi RCT," classifying all non-placebo-controlled trials as having high risk of bias, and therefore based the conclusion against VP on only the $2 N E J M$ sham trials, which were classified as Level I rather than Level II data. This arbitrary classification and selective information culling is one example of how significant discrepancies can arise between HTA reviews and high-quality meta-analyses.

Our present meta-analysis showed differences in outcomes between BKP, VP, and NSM, but fewer than seen in the Papanastassiou et $\mathrm{al}^{52}$ metaanalysis. More pain reduction for both BKP and VP were observed in comparison with NSM, although this was significantly different from BKP to NSM but not statistically significant from VP to
NSM. Our analysis also included VAI as a subgroup. We found a significant difference in VAS scores between BKP and VAI, with BKP having more reductions in pain score. BKP had some tendency for slightly better pain reduction than VP, but the difference in this meta-analysis was not statistically significant. This evaluation also showed no difference in disability improvement between BKP and VP and no difference in rates of subsequent fractures. BKP showed significantly better outcomes than VAI in terms of disability improvement (ODI). Patients undergoing BKP were found not to have significantly greater amounts of anterior and posterior vertebral height restoration than VP patients, nor was a difference seen in kyphotic angle correction between VP and BKP groups.

Along with the various outcome measures, a few studies in this meta-analysis examined economic costs of BKP and VP. Folman and Shabat ${ }^{32}$ concluded that BKP and VP had similar success levels of pain relief, but due to higher costs of BKP, the cost-benefit advantage of VP was clear. However, though an analysis of Medicare data showed no differences in adjusted, cumulative treatment costs for VP and BKP patients in the first 9 months postsurgery, BKP patients were subsequently associated with significantly lower adjusted treatment costs in remaining periods through 2 years postsurgery. ${ }^{56}$ A survival and cost analysis by Lange et $\mathrm{al}^{12}$ comparing BKP and VP using German claims data showed 4-year mean overall costs were lower for the BKP group than for VP (BKP: $€ 39014$ versus VP: $€ 42510$ ), due to higher initial BKP costs being offset by pharmacy costs in VP patients. In a multicenter, randomized, controlled cost-effectiveness analysis, Fritzell et $\mathrm{l}^{33}$ determined that it was not possible to demonstrate that BKP was cost effective compared with standard medical treatment in VCF patients. Three other studies (Ström et al, ${ }^{57}$ Svedbom et $\mathrm{al}^{58}$ and Klazen et $\mathrm{al}^{59}$ ) analyzed cost effectiveness using quality adjusted life years and confirmed vertebral augmentation was cost effective compared with NSM.

In a comparison of BKP and VBS, Werner et $\mathrm{al}^{15}$ found no substantial differences in cost, even though costs of vertebral fracture treatment were generally lower for BKP than VBS for treating 1 to 3 vertebral levels.

This meta-analysis yielded insufficient data to determine the QOL differences and optimal inter- 
vention time relative to age of the $\mathrm{VCF}$. There were also insufficient data to determine differences in extravasation rates or serious adverse events. We demonstrated comparable findings to those of a recent meta-analysis comparing NSM with VP by Mattie et al. ${ }^{60}$ When evaluating 11 studies containing 1048 participants, Mattie et $\mathrm{al}^{60}$ reported VP was better than NSM with respect to pain relief up to 1 year after VP. This provides additional information supportive of the efficacy of VP, similar to previous meta-analyses by Anderson et $\mathrm{al}^{53}$ and Papanastassiou et $\mathrm{al}^{52}$ and trends shown in several of the 25 articles included in this meta-analysis. The level of evidence of the meta-analysis of Mattie et $\mathrm{al}^{60}$ was designated as Oxford Level I, which should be taken into account by not only treatment guidelines but also policy recommendations and HTAs. The meta-analysis also focused specifically on percutaneous vertebroplasty as compared with conservative therapy, while only evaluating studies in which the primary outcome was pain relief. No requirements were made of retrospective or prospective studies, minimum number of patients, or site of the procedure.

\section{CONCLUSION}

This meta-analysis revealed a large number of high-quality articles (25 Level I and Level II studies), including 17 randomized trials since February 2011. We were unable to include findings from a recently completed prospective, multicenter evaluation of the safety and efficacy of BKP in Medicare patients (EVOLVE trial ${ }^{61}$ ), as it had not yet been published in a peer-reviewed journal. The results of this EVOLVE trial included statistically significant improvements in patients' pain, function, quality of life, self-care indices, and vertebral deformity correction. Our current meta-analysis showed statistically superior pain reduction for BKP in comparison with NSM, but pain reduction was not statistically significant for VP in comparison with NSM. There was significantly improved pain reduction and disability improvement for BKP over VAI. BKP tended to show greater anterior and posterior vertebral height change than VP, though this was not statistically significant. The overall number of statistically significant categories was less than in a previous 2011 meta-analysis. It is noteworthy that the analysis includes a VP versus sham trial that offers Level I evidence of statistically significant differences in pain reduction and disabil- ity improvements in a study with higher statistical power than previous sham trials, which showed no difference between VP and sham. Also notable is the refinement of evidence, including reclassification of 2 previous sham trials to Level II evidence and conclusions of best-quality literature that serve to further define the safety and efficacy of vertebral augmentation. Despite the preponderance of highquality data, all endpoints demonstrated substantial heterogeneity in the treatment arms even between randomized trials.

\section{REFERENCES}

1. Baaj AA, Downes K, Vaccaro AR, Uribe JS, Vale FL. Trends in the treatment of lumbar spine fractures in the United States: a socioeconomics perspective: clinical article. J Neurosurg Spine. 2011;15(4):367-370. https://doi.org/10.3171/2011.5. SPINE10934.

2. Barr JD, Barr MS, Lemley TJ, McCann RM. Percutaneous vertebroplasty for pain relief and spinal stabilization. Spine (Phila Pa 1976). 2000;25(8):923-928.

3. Black DM, Cummings SR, Karpf DB, et al. Randomised trial of effect of alendronate on risk of fracture in women with existing vertebral fractures. Fracture Intervention Trial Research Group. Lancet. 1996;348(9041):1535-1541.

4. Melton LJ III. Adverse outcomes of osteoporotic fractures in the general population. $J$ Bone and Miner Res. 2003;18(6):1139-1141. https://doi.org/10.1359/jbmr.2003.18.6. 1139.

5. Cooper C, Atkinson EJ, O'Fallon WM, Melton JL III. Incidence of clinically diagnosed vertebral fractures: a population based study in Rochester, Minnesota, 1985-1989. J Bone Miner Res. 1992;7(2):221-227. https://doi.org/10.1002/jbmr. 5650070214 .

6. Babayev M, Lachmann E, Nagler W. The controversy surrounding sacral insufficiency fractures: to ambulate or not to ambulate? Am J Phys Med Rehabil. 2000;79(4):404-409.

7. Lau E, Ong K, Kurtz S, Schmier J, Edidin A. Mortality following the diagnosis of a vertebral compression fracture in the Medicare population. J Bone Joint Surg Am. 2008;90(7):1479-1486. https://doi.org/10.2106/JBJS.G.00675.

8. Cauley JA, Thompson DE, Ensrud KC, Scott JC, Black D. Risk of mortality following clinical fractures. Osteoporos Int. 2000;11(7):556-561. https://doi.org/10.1007/s001980070075.

9. Chen AT, Cohen DB, Skolasky RL. Impact of nonoperative treatment, vertebroplasty, and kyphoplasty on survival and morbidity after vertebral compression fracture in the medicare population. J Bone Joint Surg Am. 2013;95(19):1729-1736. https://doi.org/10.2106/JBJS.K.01649.

10. Edidin AA, Ong KL, Lau E, Kurtz SM. Mortality risk for operated and nonoperated vertebral fracture patients in the medicare population. J Bone Miner Res. 2011;26(7):617-626. https://doi.org/10.1002/jbmr.353.

11. Lange A, Kasperk C, Alvares L, Sauermann S, Braun S. Survival and cost comparison of kyphoplasty and percutaneous vertebroplasty using German claims data. Spine (Phila Pa 1976). 2014;39(4):318-326. https://doi.org/10.1097/ BRS.0000000000000135. 
12. Zampini JM, White AP, McGuire KJ. Comparison of 5766 vertebral compression fractures treated with or without kyphoplasty. Clin Orthop Relat Res. 2010;468(7):1773-1780. https://doi.org/10.1007/s11999-010-1279-7.

13. Edidin AA, Ong KL, Lau E, Kurtz SM. Morbidity and mortality after vertebral fractures: comparison of vertebral augmentation and nonoperative management in the medicare population. Spine (Phila Pa 1976). 2015;40(15):1228-1241. https://doi.org/10.1097/BRS.0000000000000992.

14. Tutton SM, Pflugmacher R, Davidian M, Beall DP, Facchini FR, Garfin SR. KAST Study: the Kiva system as a vertebral augmentation treatment - a safety and effectiveness trial: a randomized, non-inferiority trial comparing the Kiva system to balloon kyphoplasty in treatment of osteoporotic vertebral compression fractures. Spine (Phila Pa 1976). 2015;40(12):865875. https://doi.org/10.1097/BRS.0000000000000906.

15. Werner CM, Osterhoff G, Schlickeiser J, et al. Vertebral body stenting versus kyphoplasty for the treatment of osteoporotic vertebral compression fractures. J Bone Joint Surg Am. 2013;95(7):577-584. https://doi.org/10.2106/JBJS.L.00024.

16. Noriega DC, Ramajo RH, Lite IS, et al. Safety and clinical performance of kyphoplasty and SpineJack $\left({ }^{\circledR}\right)$ procedures in the treatment of osteoporotic vertebral compression fractures: a pilot, monocentric, investigator-initiated study. Osteoporos Int. 2016;27(6):2047-2055. https://doi.org/10.1007/ s00198-016-3494-X.

17. Krüger A, Oberkircher L, Figiel J, et al. Height restoration of osteoporotic vertebral compression fractures using different intravertebral reduction devices: a cadaveric study. Spine J. 2015;15(5):1092-1098. https://doi.org/10.1016/j. spinee.2013.06.094.

18. Jensen ME, McGraw JK, Cardella JF, Hirsch JA. Position statement on percutaneous vertebral augmentation: a consensus statement developed by the American Society of Interventional and Therapeutic Neuroradiology, Society of Interventional Radiology, American Association of Neurological Surgeons/Congress of Neurological Surgeons, and American Society of Spine Radiology. $J$ Neurointerv Surg. 2009;1(2):181-185.

19. Buchbinder R, Osborne RH, Ebeling PR, et al. A randomized trial of vertebroplasty for painful osteoporotic vertebral fractures. $N$ Engl J Med. 2009;361(6):557-568. https:// doi.org/10.1056/NEJMoa0900429.

20. Kallmes DF, Comstock BA, Heagerty PJ, et al. A randomized trial of vertebroplasty for osteoporotic spinal fractures. N Engl J Med. 2009;361(6):569-579. https://doi.org/ 10.1056/NEJMoa0900563.

21. Clark, W, Bird P, Gonski P, et al. Safety and efficacy of vertebroplasty for acute painful osteoporotic fractures (VAPOUR): a multicentre, randomised, double-blind, placebocontrolled trial. Lancet. 2016;388(10052):1408-1416. https:// doi.org/10.1016/S0140-6736(16)31341-1.

22. Hansen EJ, Simony A, Rousing R, Carreon LY, Tropp H, Andersen MO. Double blind placebo-controlled trial of percutaneous vertebroplasty (VOPE). Global Spine J. 2016;6(suppl 1):GO106. https://doi.org/10.1055/s-0036-1582763.

23. Endres S, Badura A. Shield kyphoplasty through a unipedicular approach compared to vertebroplasty and balloon kyphoplasty in osteoporotic thoracolumbar fracture: a prospective randomized study. Orthop Traumatol Surg Res. 2012;98(3):334-340. https://doi.org/10.1016/j.otsr.2011.11.010.
24. Korovessis P, Vardakastanis K, Repantis T, Vitsas V. Balloon kyphoplasty versus KIVA vertebral augmentationcomparison of 2 techniques for osteoporotic vertebral body fractures: a prospective randomized study. Spine (Phila $\mathrm{Pa}$ 1976). 2013;38(4):292-299. https://doi.org/10.1097/BRS. 0b013e31826b3aef.

25. Otten LA, Bornemn R, Jansen TR, et al. Comparison of balloon kyphoplasty with the new Kiva ${ }^{\circledR}$ VCF system for the treatment of vertebral compression fractures. Pain Physician. 2013;16(5):E505-E512.

26. Borgström F, Olafsson G, Ström O, et al. The impact of different health dimensions on overall quality of life related to kyphoplasty and nonsurgical management. Osteoporos Int. 2013;24(7):1991-1999. https://doi.org/10.1007/s00198-012-2237-x.

27. Hozo SP, Djulbegovic B, Hozo I. Estimating the mean and variance from the median, range, and the size of a sample. BMC Med Res Methodol. 2005;5(00):13. https://doi.org/10. 1186/1471-2288-5-13.

28. Boonen S, Van Meirhaeghe J, Bastian L, et al. Balloon kyphoplasty for the treatment of acute vertebral compression fractures: 2-year results from a randomized trial. J Bone Miner Res. 2011;26(7):1627-1637. https://doi.org/10.1002/jbmr.364.

29. Dohm M, Black CM, Dacre A, et al. A randomized trial comparing balloon kyphoplasty and vertebroplasty for vertebral compression fractures due to osteoporosis. AJNR Am J Neuroradiol. 2014;35(12):2227-2236. https://doi.org/10.3174/ ajnr.A4127.

30. Dong R, Chen L, Tang T, et al. Pain reduction following vertebroplasty and kyphoplasty. Int Orthop. 2013;37(1):83-87. https://doi.org/10.1007/s00264-012-1709-0.

31. Farrokhi MR, Alibai E, Maghami Z. Randomized controlled trial of percutaneous vertebroplasty versus optimal medical management for the relief of pain and disability in acute osteoporotic vertebral compression fractures. J Neurosurg Spine. 2011;14(5):561-569. https://doi.org/10.3171/2010.12. SPINE10286.

32. Folman Y, Shabat S. A comparison of two new technologies for percutaneous vertebral augmentation: confidence vertebroplasty vs. sky kyphoplasty. Isr Med Assoc J. 2011;13(7):394-397.

33. Fritzell P, Ohlin A, Borgström F. Cost-effectiveness of balloon kyphoplasty versus standard medical treatment in patients with osteoporotic vertebral compression fracture: a Swedish multicenter randomized controlled trial with 2-year follow-up. Spine (Phila Pa 1976). 2011;36(26):2243-2251. https://doi.org/10.1097/BRS.0b013e3182322d0f.

34. Kroon F, Staples M, Ebeling PR, et al. Two-year results of a randomized placebo-controlled trial of vertebroplasty for acute osteoporotic vertebral fractures. J Bone Miner Res. 2014;29(6):1346-1355.

35. Lee HM, Park SY, Lee SH, et al. Comparative analysis of clinical outcomes in patients with osteoporotic vertebral compression fractures (OVCFs): conservative treatment versus balloon kyphoplasty. Spine J. 2012;12(11):998-1005. https:// doi.org/10.1016/j.spinee.2012.08.024.

36. Li X, Yang $\mathrm{H}$, Tang $\mathrm{T}$, et al. Comparison of kyphoplasty and vertebroplasty for treatment of painful osteoporotic vertebral compression fractures: twelve-month follow-up in a prospective nonrandomized comparative study. J Spinal Disord Tech. 2012;25(3):142-149. https://doi.org/10. 1097/BSD.0b013e318213c113. 
37. Liu JT, Li CS, Chang CS, Liao WJ. Long-term followup study of osteoporotic vertebral compression fracture treated using balloon kyphoplasty and vertebroplasty. $J$ Neurosurg Spine. 2015;23(1):94-98. https://doi.org/10.3171/ 2014.11.SPINE14579.

38. Movrin I. Adjacent level fracture after osteoporotic vertebral compression fracture: a nonrandomized prospective study comparing balloon kyphoplasty with conservative therapy. Wien Klin Wochenschr. 2012;124(9-10):304-311. https:// doi.org/10.1007/s00508-012-0167-4.

39. Nakano M, Kawaguchi $Y$, Kimura $T$, Hirano N. Transpedicular vertebroplasty after intravertebral cavity formation versus conservative treatment for osteoporotic burst fractures. Spine J. 2014;14(1):39-48. https://doi.org/10.1016/j. spinee.2013.03.016.

40. Staples MP, Howe BM, Ringler MD, et al. New vertebral fractures after vertebroplasty: 2-year results from a randomised controlled trial. Arch Osteoporos. 2015;10:229. https://doi.org/10.1007/s11657-015-0229-0.

41. Van Meirhaeghe J, Bastian L, Boonen S, et al. A randomized trial of balloon kyphoplasty and nonsurgical management for treating acute vertebral compression fractures: vertebral body kyphosis correction and surgical parameters. Spine (Phila Pa 1976). 2013;38(12):971-983. https://doi.org/10. 1097/BRS.0b013e31828e8e22.

42. Wang CH, Ma JZ, Zhang CC, Nie L. Comparison of high-viscosity cement vertebroplasty and balloon kyphoplasty for the treatment of osteoporotic vertebral compression fractures. Pain Physician. 2015;18(2):E187-E194.

43. Yang DH, Cho KH, Chung YS, Kim YR. Effect of vertebroplasty with bone filler device and comparison with balloon kyphoplasty. Eur Spine J. 2014;23(12):2718-2725. https://doi.org/10.1007/s00586-014-3379-7.

44. Yokoyama K, Kawanishi M, Yamada M, et al. In not only vertebroplasty but also kyphoplasty, the resolution of vertebral deformities depends on vertebral mobility. AJNR $\mathrm{Am}$ J Neuroradiol. 2013;34(7):1474-1478. https://doi.org/10.3174/ ajnr.A3424.

45. Wright JG. Levels of evidence and grades of recommendations. http://www2.aaos.org/bulletin/apr05/fline9.asp. Accessed Month DD, YYYY.

46. Morrison WB, Parker L, Frangos AJ, Carrino JA. Vertebroplasty in the Unites States: guidance method and provider distribution, 2001-2003. Radiology. 2007;243(1):166170. https://doi.org/10/1148.radiol.2431060045.

47. Brett AS. Use of vertebroplasy and kyphoplasty declined in 2010. $J$ Watch General. 2013;201(00):237.

48. Long SS, Morrison WB, Parker L. Vertebroplasty and kyphoplasty in the United States: provider distribution and guidance method, 2001-2010. AJR Am J Roentgenol. 2012;199(6):1358-1364. https://doi.org/10.2214/AJR.12.8733.

49. Leake CB, Brinjikji W, Cloft HJ, Kallmes DF. Trends in inpatient spine augmentation: 2001-2008. AJNR Am J Neuroradiol. 2011;32(8):1464-1468. https://doi.org/10.3174/ ajnr.A2503.

50. Barr JD, Jensen ME, Hirsch JA, et al. Position statement on percutaneous vertebral augmentation: a consensus statement developed by the Society of Interventional Radiology (SIR), American Association of Neurological Surgeons (AANS) and the Congress of Neurological Surgeons (CNS), American College of Radiology (ACR), American Society of Neuroradi- ology (ASNR), American Society of Spine Radiology (ASSR), Canadian Interventional Radiology Association (CIRA), and the Society of NeuroInterventional Surgery (SNIS). J Vasc Interv Radiol. 2014;25(2):171-181. https://doi.org/10.1016/j.jvir. 2013.10.001.

51. Esses SI, McGuire R, Jenkins J, et al. American Academy of Orthopaedic Surgeons clinical practice guideline on: the treatement of osteoporotic spinal compression fractures. J Bone Joint Surg Am. 2011;93(20):1934-1936. https://doi.org/ 10.2106/JBJS.9320ebo.

52. Papanastassiou ID, Phillips FM, Van Meirhaeghe J, et al. Comparing effects of kyphoplasty, vertebroplasty, and nonsurgical management in a systematic review of randomized and non-randomized controlled studies. Eur Spine J. 2012;21(9):1826-1843. https://doi.org/10.1007/s00586-012-2314$\mathrm{z}$.

53. Anderson PA, Froyshteter AB, Tontz WL Jr. Metaanalysis of vertebral agumenation compared with conservative treatement for osteoporotic spinal fractures. J Bone Miner Res. 2013;28(2):372-382. https://doi.org/10.1002/jbmr.1762.

54. Bono CM, Ghiselli G, Gilbert TJ, et al. An evidencebased clinical guideline for the diagnosis and treatment of cervical radiculopathy from degenerative disorders. Spine $J$. 2011;11(1):64-72. https://doi.org/10.1016/j.spinee.2010.10.023.

55. Buchbinder R, Golmohammadi K, Johnston RV, et al. Percutaneous vertebroplasty for osteoporotic vertebral compression fracture. Cochrane Database Syst Rev. 2015;(4):CD006349. https://doi.org/10.1002/14651858.CD006349.pub2.

56. Ong KL, Lau E, Kemner JE, Kurtz SM. Two-year cost comparison of vertebroplasty and kyphoplasty for the treatement of vertebral compression fractures: are initial surgical costs misleading? Osteoporos Int. 2013;24(4):1437-1445. https:// doi.org/10.1007/s00198-012-2100-0.

57. Stöm O, Borgström F, Kanis JA, et al. Osteoporosis: burden, health care provision and opportunities in the EU: a report prepared in collaboration with the International Osteoporosis Foundation (IOF) and the European Federation of Pharmaceutical Industry Associations (EFPIA). Arch Osteoporos. 2011;6(1-2):59-155. https://doi.org/10.1007/s11657-0110060-1.

58. Svedbom A, Alvares L, Cooper C, Marsh D, Ström O. Balloon kyphoplasty compared to vertebroplasty and nonsurgical management in patients hospitalised with acute osteoporotic vertebral compression fracture: a UK cost-effectiveness analysis. Osteoporos Int. 2013;24(1):355-367. https://doi.org/10. 1007/s00198-012-2102-y.

59. Klazen CA, Lohle PN, de Vries J, et al. Vertebroplasty versus conservative treatment in acute osteoporotic vertebral compression fractures (Vertos II): an open-label randomised trial. Lancet. 2010;376(9746):1085-1092. https://doi.org/10. 1016/S0140-6736(10)60954-3.

60. Mattie R, Laimi K, Yu S, Saltychev M. Comparing percutaneous vertebroplasty and conservative therapy for treating osteoporotic compression fractures in the thoracic and lumbar spine: a systematic review and meta-analysis. $J$ Bone Joint Surg Am. 2016;98(12):1041-1051. https://doi.org/10. 2106/JBJS.15.00425.

61. Beall DP, Chambers MF, Thomas SM, et al. EVOLVE: A prospective multicenter evaluation of quality of life, pain \& activities of daily living outcomes for balloon kyphoplasty in the treatment of medicare patients with vertebral compression 
fractures. Evidence-Based Spine Interventions Seminar, Palm Springs, CA, January 27-28, 2017.

Disclosures and COI: This meta-analysis was conducted with funding from the International Society for the Advancement of Spine Surgery (ISASS); Benvenue Medical, Inc; DePuy Synthes Spine, a division of DePuy Orthopaedics, Inc; DFINE Inc; Globus Medical, Inc; Medtronic Sofamor Danek USA, Inc; and Stryker. Douglas Beall reports the following disclosures unrelated to the preparation of this manuscript: compensation, grants, and personal fees from Medtronic related to consultancy, research and teaching; grants from Benvenue, Alphatech Spine, Medical Metrics, Liventa, Vexim, and Mesoblast; consultancy fees from DEFINE and Osseon; other from Lilly, Smith \& Nephew, Biomet, Vertiflex, Synthes, and Integral Spine Solutions. Morgan Lorio reports the following disclosures unrelated to the preparation of this manuscript: royalties from Choice Spine Thunderbolt, stock ownership in Orthogenerex, stock options with OrthoKinematics, intellectual property with Transformer Spine, investigator for Acadia Facet IDE Trial. B. Min Yun and Maria J. Runa are employees of Exponent, a scientific and engi- neering consulting firm. They have no other disclosures. Kevin Ong reports the following disclosures: employee and shareholder of Exponent, a scientific and engineering consulting firm. Exponent has been paid fees by entities in the biomedical arena for consulting services, including Zimmer Biomet, Stryker Orthopaedics, Joerns Healthcare, Ossur, DJO, Ethicon, Ferring Pharmaceuticals, Pacira Pharmaceuticals, Paradigm Spine, and Medtronic. Christopher B. Warner reports no disclosures.

Corresponding Author: Christopher B. Warner, MD, University of Colorado Anschutz Medical Campus, Department of Radiology, Mail Stop 8200, AO1, Rm 2414, 12631 East 17th Ave, Aurora, CO 80045. Phone: (000) 000-0000; Email: Christopher.Warner@ucdenver.edu.

Published 15 August 2018

This manuscript is generously published free of charge by ISASS, the International Society for the Advancement of Spine Surgery. Copyright $\subset 2018$ ISASS. To see more or order reprints or permissions, see http://ijssurgery.com. 